\title{
A dynamic subfilter-scale model for plane parallel flows
}

\author{
B. Dubrulle \\ NCAR, P.O. Box 3000, Boulder, Colorado 80307-3000 \\ and CNRS, URA 285, Observatoire Midi-Pyrénées, 14 avenue Belin, F-31400 Toulouse, France
}

J. P. Laval

NCAR, P.O. Box 3000, Boulder, Colorado 80307-3000

and SAp/DAPNIA/DSM/CEA, CE Saclay, 91191 Gif sur Yvette Cedex, France

S. Nazarenko

Mathematics Institute, University of Warwick, Coventry CV4 7AL, United Kingdom

N. K.-R. Kevlahan

Department of Mathematics and Statistics, McMaster University, Hamilton, Ontario L8S 4K1, Canada

(Received 13 July 1999; accepted 19 April 2001)

\begin{abstract}
We present a dynamic model of the subfiltered scales in plane parallel geometry using a generalized, stochastic rapid distortion theory (RDT). This new model provides expressions for the turbulent Reynolds subfilter-scale stresses via estimates of the subfilter velocities rather than velocity correlations. Subfilter-scale velocities are computed using an auxiliary equation which is derived from the Navier-Stokes equations using a simple model of the subfilter energy transfers. It takes the shape of a RDT equation for the subfilter velocities, with a stochastic forcing. An analytical test of our model is provided by assuming delta-correlation in time for the supergrid energy transfers. It leads to expressions for the Reynolds stresses as a function of the mean flow gradient in the plane parallel geometry and can be used to derive mean equilibrium profiles both in the near-wall and core regions. In the near-wall region we derive a general expression for the velocity profile which is linear in the viscous layer and logarithmic outside. This expression involves two physical parameters: the von Karman constant and the size of the viscous layer (which can be computed via a numerical implementation of our model). Fits of experimental profiles using our general formula provides reasonable values of these parameters $(\kappa=0.4$ to $\kappa=0.45$, the size of the viscous layer is about 15 wall units). In the core region, we find that the shape of the profile depends on the geometry of the flow; it ranges from algebraic in channel flow, to exponential in the bulk of boundary layers, and linear in plane Couette flow. This classification is consistent with Oberlack's system, which is based on symmetry arguments. Fits of boundary layer flows or channel flows at different Reynolds number over the whole flow region are performed using our results, and are found to be in very good agreement with available data. () 2001 American Institute of Physics. [DOI: $10.1063 / 1.1378038]$
\end{abstract}

\section{INTRODUCTION}

Turbulent shear flows are widespread and their structure has been investigated extensively for many decades in laboratory and numerical experiments. It was commonly accepted that the mean velocity profile across the channel is piecewise; a linear "viscous profile" near the wall, a logarithmic profile away from the wall, followed by an algebraic profile $^{1}$ in the center of the channel. This description appeared to be supported by a simple dimensional analysis due to von Karman and by several matched asymptotic theories (see, e.g., Refs. 2 and 3) which all led to the famous log law of the wall under appropriate asymptotic limits.

More recently, a controversy arose when Barenblatt ${ }^{4}$ used a dimensional argument combined with an assumption of incomplete similarity to obtain a family of power laws, with logarithmic envelope, as the description of the mean velocity profile in a channel. In his theory, the index of the power law varies with the Reynolds number. This depen- dence was subsequently detected in the high Reynolds number data of Nikuradze, ${ }^{5}$ but found to be weak, like $1 / \ln (\mathrm{Re})$. As a consequence, mean velocity profiles measured at different Reynolds number tend to scatter slightly and reveal only the envelope of the family of curves, i.e., the log-law. This controversy reached a climax when a team from Princeton used measurements made at the Super Pipe facility to test the Barenblatt theory. The Reynolds numbers obtained in this configuration are quite large, even larger than those obtained in the experiment of Nikuradze. The final result apparently contradicted Barenblatt's theory, and was more consistent with the log-law, preceded by an algebraic profile with Reynolds independent index. ${ }^{6}$ Barenblatt and Chorin have reanalyzed these data, and claim to have found a roughness dependence of the result which makes them less suitable to test their theory than the Nikuradze data.

Another recent theory for turbulent pipe/channel and boundary layer flow has been developed by George et al. ${ }^{7,8}$ This theory is also based on dimensional analysis and 
matched asymptotic expansions, however this group bases their analysis directly on the Reynolds-averaged NavierStokes equations. They determined that the correct velocity to scale the outer region is $U_{\infty}$, while the friction velocity $u_{*}$ should be used to scale the inner region near the wall. Both regions follow Reynolds number dependent power laws, but with different constants and origins. However, they found that the Reynolds number dependence should be much stronger for a zero-pressure gradient boundary layer than for pipe and channel flows (where Reynolds number dependence is negligible). Note that they assume the same Reynolds number dependence as Barenblatt. The two different forms for the inner and outer scaling imply the existence of a mesolayer in which dissipative scales are not fully separated from the energy and Reynolds stress producing ones. The mesolayer follows a power law in boundary layers and a logarithmic law in pipe and channel flow. In both cases the profiles are functions of $y+a$ rather than $y$ alone (where $a$ is an arbitrary constant). Their theory also matches the Super Pipe data very well.

At the same time new results were obtained which only added to the confusion. Oberlack ${ }^{9}$ used a Lie group analysis of the equations of motion to classify all the possible equilibrium velocity profiles allowed by the symmetry of the Navier-Stokes equation. The results include the logarithmic law, the algebraic law, the viscous sublayer, the linear profile and the exponential profile as particular cases. This study was followed by a data analysis to try to determine the actual conditions required for each law to be realized. Analyzing Niederschulte's data, ${ }^{10}$ Oberlack found evidence of an algebraic law extending almost to the center of the channel, and scaling with the channel's half-width instead of with the "inner scale" (viscous scale) predicted in Barenblatt's theory. Moreover, the index of the power law measured by Oberlack was significantly larger (one order of magnitude) than that predicted by the theory. An analysis of the Super Pipe data of Zagarola ${ }^{11}$ confirmed the existence of this algebraic law covering $80 \%$ of the center of the pipe. ${ }^{12}$ More recently, the same data have been shown to fit a modified cosh-profile arising from a new closure equation. ${ }^{13}$ Since all the theories describing these results can be put on the same phenomenological (dimensional or closure-like) footing, it is hard to decide which is right and in which cases. In a previous paper, ${ }^{14}$ we derived the equilibrium velocity profile for a flow in the near-wall region using a WKB method generalizing rapid distortion theory, using a simplified assumption about the generation of small-scales. The main elements of this approach can be most easily explained using the simplest example of two-dimensional shear flow turbulence (see Refs. 15 and 32). Specifically, we modeled the debris of the nearwall vortices penetrating the outer regions as a weak external forcing acting at small-scales. This simplification allows one to assume nonlocality, e.g., to neglect interactions between the small scales in comparison with their interactions with a well separated in scale mean flow. In turn, this allows exact analytical solutions, with the celebrated log law of the wall being one of them in the case of a forcing with short correlation in time and statistically homogeneous in space.

Note that the role of nonlocal and local interactions in wall bounded turbulence is related to an old and yet unresolved controversy about applicability of RDT to such turbulence. Recent numerical simulations suggest that even for isotropic 3D turbulence, a combination of RDT and a scale dependent eddy viscosity provides a realistic description of both the small-scale spectra and the higher cumulants related with intermittency ${ }^{16}$ In wall bounded flows, the presence of the strong mean makes interaction of small scales even more nonlocal and dominating over the eddy viscosity effect. Recent numerical and new experimental evidence of nonlocality in such turbulence was reported in Refs. 17 and 18. However, our understanding of the role of nonlocality is still far from being complete and the approach developed in Refs. 14 and 15, and the present paper should be treated as a model, rather than a rigorously validated theory.

From a theoretical point of view, the approach developed in Ref. 14 is interesting because it suggests a way of approaching the mean profile controversy which is not based on dimensional arguments and similarity assumptions about the solution of the Navier-Stokes equations, but rather on the statistical properties of the energy transfers between large and small scales. In this respect, the work described in Ref. 14 is incomplete, because it only characterizes the conditions for existence of the log and algebraic laws, and does not relate the realizability of these conditions to the geometry of a particular flow. Thus, it does not specify where these various profiles should be expected (e.g., near the wall, in the core region or in the outer region). A basic fact therefore remains unexplained: why does the shape of the equilibrium profile change from a log-law near the wall ${ }^{19}$ to an algebraic or exponential ${ }^{9}$ defect law further away? This transition probably reflects some changes in the generation of the small-scales which needs to be taken into account by a selfconsistent model of the forcing. The purpose of the present paper is to derive such a self-consistent model of channel flow by making two modifications of the WKB-RDT model of Ref. 14. These changes make it suitable for future use in numerical large eddy simulations (LES).

First, we replace the statistical averaging procedure of the previous paper with space averages, which are more suitable for numerical and laboratory experiments. Indeed, in numerical simulation, one either performs horizontal averages over the streamwise directions, or spatial average over a given scale, representing the smallest resolved scale in the simulation (large eddy simulation approach); in laboratory experiments one usually performs time averages which are then translated into spatial averages using Taylor's hypothesis. In contrast, statistical averages require a large number of realizations of the same flow, which, although useful theoretically, is often impractical.

The second new element of our model is that the external forcing is chosen based on physical requirements, such as zero turbulent stress at the wall, and takes exactly into account the supergrid energy transfers. The complete model is described in Sec. II.

In a second part (Secs. III and IV), we test the physical soundness of our model by computing the equilibrium profiles within the whole domain under the approximation of delta-correlation in time for the supergrid energy transfers, 
like in the WKB-RDT model. By moving from this model with external forcing to our LES-RDT model with selfconsistent forcing, we give up the possibility of exact analytical solutions because of the nonlinear dynamical coupling. We show here that some simple physical assumptions lead to a solution where the subfilter Reynolds stresses can be expressed as functions of the resolved quantities and some numerical constants (which cannot be derived analytically). Thus, the corresponding set of closed equations for the resolved velocities can be integrated further analytically, or semianalytically, to find qualitatively the equilibrium profile in any part of the channel. We find that the qualitative profile changes from a log-law near the boundary, to an algebraic (exponential) defect law for a flow within a closed channel (over a flat plate).

Let us be aware however that the practicality of the space average comes at expense of the theoretical complexity (the mean of the mean is not equal to the mean, etc.). In particular there will be greater variety of terms corresponding to the turbulent stresses to describe which one has to sacrifice rigor and resort to simplified models. Therefore, the present paper is not intended to be as fully rigorous as the previous one. We allow ourselves the liberty, when necessary, of making simplifying assumptions which are not fully justified. We will attempt to minimize the arbitrariness of our assumptions by providing technical or mathematical justifications where possible. However, to simplify the paper, we have placed the technical details of the derivation in the Appendices.

\section{THE DYNAMIC MODEL}

\section{A. Notations and constitutive equations}

We consider an incompressible velocity field $\mathbf{u}(\mathbf{x}, t)$ satisfying the Navier-Stokes equations,

$$
\begin{aligned}
& \partial_{i} u_{i}=0, \\
& \partial_{t} u_{i}+\partial_{j}\left(u_{i} u_{j}\right)=-\partial_{i} p+\nu \Delta u_{i}+\sigma_{i},
\end{aligned}
$$

where $p$ is the pressure, $\nu$ is the kinematic viscosity, and $\sigma$ is an external forcing. Note that for the pressure driven flows (channel, pipe) the external pressure gradient is included in $\sigma$ (rather than $\nabla p$ ) in our notations. We have set the (constant) density equal to one for simplicity.

In this paper we shall consider two types of geometry: plane channel flow (where the flow is bounded by two infinite flat rigid plates separated by a vertical distance $2 d$ ) and a flat plate boundary layer (where the fluid is parallel to and bounded by a single flat plate). In the first case, the forcing is via a constant pressure gradient applied in the $x$ direction parallel to the plates $\sigma=\left(-d P_{*} / d x ; 0,0\right)$, or by constant movement of plates in opposite directions (plane Couette flow). In the second case, we assume no external forcing $(\sigma=0)$, but we allow for a free stream velocity $u_{\infty}$ at the outer (infinite) edge of the boundary layer. We assume noslip boundary conditions for the velocity on the rigid plates.

\section{B. Resolved scale equations}

To derive the large-scale equations, we decompose the fields into their resolved and subfilter parts

$$
\mathbf{u}=\overline{\mathbf{u}}+\mathbf{u}^{\prime} \equiv \mathbf{U}+\mathbf{u}^{\prime},
$$

where the average is taken via a filter [which is defined and discussed in Appendix A; see Eq. (A3)]. Note that from now on we shall refer to $\bar{u}$ as the resolved velocity, as distinguished from the mean velocity $\langle u\rangle$ which is obtained by averaging the velocity horizontally and in time. Applying this decomposition to the Navier-Stokes equations (1) and taking into account the fact that the averaging and the $y$-component of the Laplacian for the horizontal velocity do not commute (see Appendix A), we obtain the following equation for the resolved streamwise velocity:

$$
\partial_{t} U_{i}+\partial_{j} \overline{u_{i} u_{j}}=-\partial_{i} P+\nu \Delta U_{i}+\mathcal{S}(z) \delta_{i 1}+\overline{\sigma_{i}} .
$$

Here, $\mathcal{S}(z)$ are the surface terms, due to the boundary conditions. These surface terms depend on the geometry of the flow

$$
\begin{aligned}
\mathcal{S}_{\text {channel }}= & u_{*}^{2}\left(g_{3}(z-d)+g_{3}(z+d)\right), \\
\mathcal{S}_{\text {Couette }}= & u_{*}^{2}\left(g_{3}(z-d)-g_{3}(z+d)\right) \\
& +\nu U_{0}\left(\frac{d g_{3}}{d z}(z-d)-\frac{d g_{3}}{d z}(z+d)\right), \\
\mathcal{S}_{\mathrm{BL}}=- & u_{*}^{2}\left(g_{3}(z)\right)+\nu u_{\infty} \frac{d g_{3}}{d z}(z-h) .
\end{aligned}
$$

Here, $h$ is the height of the boundary layer and $U_{0}$ is the speed of the plates for the plane Couette flow $\left(U= \pm U_{0}\right.$ at $z=\mp d)$. The function $g_{3}(z)$ is the correction due to the filter which depends explicitly on the filter choice and on the cutoff scale in the vertical direction.

If we use the decomposition (2), we can divide the nonlinear terms of (3) into several parts,

$$
\begin{aligned}
\partial_{t} U_{i} & +\partial_{j} \overline{U_{i} U_{j}}+\partial_{j}\left\langle\overline{U_{i} u_{j}^{\prime}+U_{j} u_{i}^{\prime}}\right\rangle+\partial_{j} \overline{u_{i}^{\prime} u_{j}^{\prime}} \\
& =-\partial_{i} P+\nu \partial_{j j} U_{i}+\mathcal{S}(z) \delta_{i 1}+\overline{\sigma_{i}} .
\end{aligned}
$$

The resolved scale motions depend on the subfilter scales via two terms: the usual Reynolds stresses, involving only subfilter-scale quantities, and an unusual cross term between subfilter and resolved quantities. Traditional LES usually models these two terms together. However, recent numerical experiments by Domaradzki et al. ${ }^{17}$ indicate that in a channel flow, these two terms have very different influence on the mean flow: the subfilter-subfilter term appears to be about $50 \%$ smaller than the subfilter-resolved component. In the sequel of the paper, we show that we are able to model the two terms separately, and hence are able to better capture their different properties by computing them via the evolution of the subfilter-scale velocities. This evolution is given by subfilter-scale equations.

\section{Subfilter-scale equations}

To find the subfilter-scale equations, we consider the subfilter component of the constitutive Eqs. (1), 


$$
\begin{aligned}
& \partial_{i} u_{i}^{\prime}=0, \\
& \partial_{t} u_{i}^{\prime}+\partial_{i}\left(u_{i} u_{j}-\overline{u_{i} u_{j}}\right)=-\partial_{i} p^{\prime}+\nu \Delta u_{i}^{\prime}-\mathcal{S}(z) \delta_{i 1} .
\end{aligned}
$$

Note that the subfilter part of the (constant) external forcing $\sigma$ is zero. At this level, we see that the small scales are generated via two types of terms: the subfilter part of the nonlinear cascade from large to small scales [as expressed by the second term of the lhs of (6)], and the surface term. This is consistent with the physics of the problem: small-scale turbulence is generated at the surface or by a transfer of energy to smaller scales (termed forward scatter).

Now we can use decomposition (2) to split the nonlinear part of (6) into terms involving resolved-subfilter-scale products and subfilter-subfilter-scale products (the resolvedresolved-scale term is zero because of the plane parallel geometry). The relative magnitude of these terms has been estimated by Domaradzki et al. ${ }^{17}$ and Hersant et al. ${ }^{20}$ in a low Reynolds number numerical simulation of channel flow, and by Carlier et al. ${ }^{18}$ in a high Reynolds number experimental boundary layer. They found that the subfiltersubfilter energy transfer at subfilter-scales is between one and two orders of magnitude smaller than the energy transfer induced by subfilter-resolved (nonlocal) interactions. For this reason it is reasonable to retain the nonlocal interactions exactly in the small-scale equation and to model the subfiltersubfilter terms via a turbulent viscosity. The corresponding equations are our subfilter-scale model (see below). This model has been validated in 2D and 3D homogeneous turbulence $^{21}$ by comparison with direct numerical simulations.

Retaining only the nonlocal interactions, our subfilterscale model becomes

$$
\begin{aligned}
& \partial_{j} u_{j}^{\prime}=0, \\
& \partial_{t} u_{i}^{\prime}+U_{j} \partial_{j} u_{i}^{\prime}+u_{j}^{\prime} \partial_{j} U_{i}=-\partial_{i} p^{\prime}+\left(\nu+\nu_{t}\right) \Delta u_{i}^{\prime}+F_{i},
\end{aligned}
$$

where $\nu_{t}$ is the turbulent viscosity, which needs to be prescribed to close the model. In 2D, we have shown that the energy condensation at large scale renders small scale interaction nonlocal and, therefore, $\nu_{t}$ can be taken as 0 . In plane parallel geometry there is no energy condensation, but the presence of a strong mean flow also induces substantial nonlocal effects, e.g., changing the slope of the energy spectrum from $-5 / 3$ to $-1 .^{22}$ In the sequel, we therefore adopt $\nu_{t}$ $=0$, to keep the number of free parameters in our analytical solutions as small as possible. This is not necessary, however, if this model is used for numerical simulations. In this case, one may even allow $\nu_{t}$ to be scale dependent, as was done in Ref. 16 for the case of isotropic 3D turbulence.

Note that this model is in fact an auxiliary equation for the subfilter velocities. It enables us to obtain an expression for the Reynolds stresses via a direct estimate of velocities rather than velocity correlations, as is usual. This idea was used for the first time in Ref. 23. In this work, the subfilter velocities are computed from the resolved velocities by requiring that they should coincide after suitable filtering. Even though this procedure is in the spirit of the dynamic model of
Bardina et al. ${ }^{24}$ it contains no kinetic information regarding the interactions between resolved and small scales. This information is contained in the Navier-Stokes equation, which we use as an input of our estimate procedure. Also, note that our procedure is derived directly from the Navier-Stokes equations, in contrast to $K-\epsilon$ models, which also involve the solution of an auxiliary equation.

Note also that the plane parallel geometry does not preclude horizontal variation of the subfilter-scale quantities, this creates "pseudoforces" $F_{i}$,

$$
F_{i}=-\mathcal{S}(z) \delta_{i 1}+\partial_{j}\left(\overline{U_{i} U_{j}}-U_{i} U_{j}\right)+\partial_{j} \overline{U_{j} u_{i}^{\prime}}+\partial_{j} \overline{U_{i} u_{j}^{\prime}} .
$$

We call them pseudoforces because they depend linearly on the averaged part of the subfilter-scale motions. We will eventually simplify them by replacing them with prescribed external forces, in order to get analytical solutions.

Equation (8), although superficially complicated, has a simple structure; it is a linear inhomogeneous equation for the subfilter motion. The forcing comes from the subfilter part of the nonlinear cascade amongst the resolved scales of motion. This forcing is internal in the sense that it describes the momentum transfer between the resolved and the subfilter fluid components which is not caused by an external pressure gradient. The linearity of such a forcing is the essential simplification which makes it possible in certain cases to solve the subfilter model analytically in terms of the resolved quantities. By substituting the solution back into the largescale equation, we obtain a closed problem for the resolved scales.

To take advantage of small-scale inhomogeneity (e.g., turbulent spots or streaks), we now decompose the smallscale equation into its Gabor modes (which are localized in both wave number $\mathbf{k}$ and position $\mathbf{x}$ ). The mathematical definition of the Gabor transform is given in Appendix B 1. We now take the Gabor transform of Eq. (7) and use (B5). To leading order in the scale separation (see Ref. 14 for rigorous justifications) and dropping primes for simpler notations (this means that from now on, we denote average quantities by capital letters, and fluctuating quantities by lower case letters) we obtain the following equations:

$\partial_{j} \hat{u}_{j}=0$

$$
\begin{aligned}
\partial_{t} \hat{u}_{i} & +U_{j} \partial_{j} \hat{u}_{i}+\partial_{j}\left(U_{m} k_{m}\right) \partial_{k_{j}} \hat{u}_{i} \\
& =-i k_{i} \hat{p}-\hat{u}_{j} \partial_{j} U_{i}-\nu \mathbf{k}^{2} \hat{u}_{i}+\hat{F}_{i},
\end{aligned}
$$

where $\mathbf{k}=\left(k_{x}, k_{y}, k_{z}\right)$ is the wave number. Note that in the Gabor transformation, we do not take into account surface terms because we chose for simplicity zero boundary conditions at the boundary for all subfilter quantities (for both the velocity and its derivative). Other choices of boundary conditions could lead to additional surface terms, which would be important only near boundary (more precisely at a distance from the boundary less than the typical scale of the function $f$ ).

The set of Eqs. (9) can be put into the form of a set of ray equations by introducing the total derivative, 


$$
D_{t} \equiv \partial_{t}+U_{j} \partial_{j}-\partial_{j}\left(U_{m} k_{m}\right) \partial_{k_{j}},
$$

which is a time derivative along rays $\mathbf{k}(t)$ and $\mathbf{x}(t)$,

$$
\begin{aligned}
& \dot{x}_{i}=U_{i}, \\
& \dot{k}_{i}=-k_{j} \partial_{i} U_{j} .
\end{aligned}
$$

These ray equations describe the motion of wave packets advected passively in position and wave number by a largescale flow. Using the incompressibility to eliminate the pressure, we can finally re-express the system (9) as

$$
D_{t} \hat{u}_{i}=\left(\frac{2 k_{i} k_{m}}{\mathbf{k}^{2}}-\delta_{i m}\right) \hat{u}_{j} \partial_{j} U_{m}-\nu \mathbf{k}^{2} \hat{u}_{i}+\hat{F}_{i}^{\perp},
$$

where $F_{i}^{\perp}$ are the divergence free components of the force,

$$
F_{i}^{\perp}=\left(\delta_{i m}-\frac{k_{i} k_{m}}{\mathbf{k}^{2}}\right) F_{m} .
$$

The set of Eqs. (5) and (12), where the Reynolds stresses are computed using (B6) constitute the main equations of our subfilter-scale model. This model is dynamical because it allows a time and space evolution of the Reynolds stress. It has been derived directly from the Navier-Stokes equations under the approximation of nonlocality of the supergrid energy transfer. Note that this subfilter-scale modeling is in the spirit of a recent model by Domaradzki and Saiki ${ }^{19}$ in which the subfilter velocity (rather than energy tensor) is estimated which permits the detailed computation of all terms appearing in the Reynolds stresses. Note also that because we have used the Gabor transform (as opposed to the Fourier transform), the subfilter-scale energy spectrum can vary in space, which allows for inhomogeneous turbulence.

Leaving the numerical implementation of this model to further work, we focus here on possible analytical test of our model. Indeed, there is no guarantee that our model will naturally lead to realistic mean shear flow profiles. By analytical integration, we show that our model automatically leads to shapes which are consistent with experimental data and theoretical analysis based on the symmetry of the Navier-Stokes equations.

Technically, the possibility of analytical derivation arises because after time and horizontal averaging, the large scale velocity profile is parallel to the plate ( $x$-direction), and depends only on the direction perpendicular to the plate ( $z$-direction): $\langle\mathbf{u}\rangle=(U(z), 0,0)$. (This implies a clever choice of the filtering, which is discussed in Appendix C.) In such a simple situation, the equations of motions become solvable analytically if the forces are prescribed.

Note that boundary layer flows are not strictly parallel. However, since the streamline curvature is usually very small, the flow may be considered to be locally parallel. Also, Oberlack ${ }^{9}$ showed that it is possible to take into account the streamwise dependence of the mean velocity profile by using as an outer length scale the Rotta-Clauser length $\Delta=\int_{0}^{\infty}\left(\left\langle u_{\infty}\right\rangle-\langle u\rangle\right) / u_{*} d z$, where $\langle u\rangle$ is the mean velocity and $u_{*}$ is the friction velocity, given by $\nu \partial_{z} u(z=0)$ $=u_{*}^{2}$ and $\left\langle u_{\infty}\right\rangle$ is the mean velocity at the outer edge of the boundary layer.

\section{EQUILIBRIUM PROFILES IN THE DELTA- CORRELATED APPROXIMATION}

\section{A. The time and horizontally averaged equations}

From now on, we concentrate on the derivation of equilibrium profiles in a plane parallel geometry. Namely, we wish to derive the possible shapes for $\left\langle U_{i}\right\rangle=(\langle U\rangle(z), 0,0)$, where now \langle\rangle refers to a horizontal and time average. The horizontal averaging can be performed easily in our LES formalism by taking constant functions as filters in the horizontal direction [i.e., $g_{1}(x)=g_{2}(y)=1 / L$, where $L$ is the horizontal scale over which the horizontal average is performed]. Then, $\langle U\rangle$ can be obtained easily by taking only a time average of the corresponding resolved velocity $U$. So, from now on, we use constant horizontal filters, and consider that \langle\rangle denotes a time average. In order to obtain true parallel geometry, long time averages are necessary. Note that largescale fluctuations in the mean profile (e.g., bursting events) are taken into account by the forcing term, i.e., in the term $\partial_{j}\left(\overline{U_{i} U_{j}}-U_{i} U_{j}\right)$. These fluctuations are large scale (and so cannot be combined with the turbulence fluctuations), but are an extra source of vorticity and thus can be added to the 3D forcing. The fluctuations are largely due to ejections or bursting from the boundary layer and are thus highly intermittent. We therefore assume delta-correlation in time. Note that this assumption is only required for deriving the mean profiles.

The equations for the mean profile can be obtained via a time averaging of (5),

$$
\begin{aligned}
& \partial_{z}\langle\overline{U w}\rangle+\partial_{z}\langle\overline{u w}\rangle=-\partial_{x} P+\nu \partial_{z z} U+\overline{\sigma_{x}}+\mathcal{S}(z), \\
& \partial_{z}\langle\overline{v w}\rangle=0, \\
& \partial_{z}\left\langle\overline{w^{2}}\right\rangle=-\partial_{z} P .
\end{aligned}
$$

Here, we have taken into account the plane parallel geometry, and the fact that, for constant horizontal averages, terms involving horizontal derivatives vanish. The first equation of (14) gives the mean profile. The second is a consistency equation for the subfilter stresses, to avoid the generation of spanwise mean velocity. The third equation expresses the hydrostatic equilibrium in the vertical direction.

The subfilter Reynolds stresses can be found by considering the ray equations (15) in a plane parallel geometry. They are

$$
\begin{aligned}
& D_{t} \hat{u}=\frac{k_{x}^{2}-k_{y}^{2}-k_{z}^{2}}{\mathbf{k}^{2}} \hat{w} \partial_{z} U-\nu \mathbf{k}^{2} \hat{u}+\hat{F}_{u}^{\perp}, \\
& D_{t} \hat{v}=\frac{2 k_{x} k_{y}}{\mathbf{k}^{2}} \hat{w} \partial_{z} U-\nu \mathbf{k}^{2} \hat{v}+\hat{F}_{v}^{\perp}, \\
& D_{t} \hat{w}=\frac{2 k_{x} k_{z}}{\mathbf{k}^{2}} \hat{w} \partial_{z} U-\nu \mathbf{k}^{2} \hat{w}+\hat{F}_{w}^{\perp} .
\end{aligned}
$$

The corresponding ray equations are in this geometry, 


$$
\begin{aligned}
& D_{t} x=U, \\
& D_{t} y=D_{t} z=0, \\
& D_{t} k_{x}=D_{t} k_{y}=0, \\
& D_{t} k_{z}=-k_{z} \partial_{z} U .
\end{aligned}
$$

\section{B. The internal forcing}

In general, the ray equations (11) and (12) have no simple analytical solution because the motion of the small and large scales are implicitly nonlinearly coupled via the forces; these forces determine the behavior of the small scales, but they depend on both large and small scales in a nontrivial way. This means that equilibrium solutions can in general only be found numerically, by a recursive procedure. The situation is much simpler if one allows an a posteriori characterization of the forces, by considering them as some sort of external noise, with simple prescribed statistics, or, in our case, simple time averaging properties. This idea was already used in the previous paper. ${ }^{14}$

Therefore, from now on we consider the forces $F_{u}, F_{v}$, and $F_{w}$ appearing in (7) and (9) to be external forces, with prescribed averaging properties. The simplest choice we can make is to assume that the forces are $\delta$-correlated in time. The idea is that because of Taylor's hypothesis space averages are, loosely speaking, equivalent to time averages. Then, because of the chaotic nature of the flow, solutions with different initial conditions have very different behavior. Thus, it seems reasonable to assume that the average of the product of two quantities starting at different times is zero. We shall refer to this as the $\delta$-correlation assumption. In Ref. 14 it is shown that this property allows us to write the correlation of two Gabor transforms corresponding to rays starting from same origin as

$$
\left\langle\hat{F}_{i}^{\perp}(\mathbf{x}, \mathbf{k}, t) \hat{F}_{j}^{\perp}\left(\mathbf{x}, \mathbf{k}^{\prime}, t^{\prime}\right)\right\rangle \propto A_{i j}(\mathbf{k}) \delta\left(\mathbf{k}^{\prime}+\mathbf{k}\right) \delta\left(t-t^{\prime}\right),
$$

where the coefficient of proportionality depends only on the mean gradients and on the angle between the vector components of $k$. When the internal forcing is isotropic, one may assume that the function $A_{i j}$ takes the following standard form:

$$
A_{i j}(\mathbf{k})=\frac{3 F(k)}{8 \pi k^{4}}\left(k^{2} \delta_{i j}-k_{i} k_{j}\right)
$$

Isotropy can be safely assumed in the core region, which is the region we are most interested in. It is not expected to hold near the wall. Furthermore, we shall assume that the forcing is symmetric with respect to $y \rightarrow-y$ (see Ref. 14 and below).

Since the forces are due to the energy cascade from large to small scale, it also seems reasonable to assume that they are correlated with the resolved velocity field. Using the $\delta$-correlation assumption, we show in Appendix D that the Gabor transforms of the resolved velocity field and the forces obey

$$
\begin{aligned}
& \left\langle\hat{U}(\mathbf{x}, \mathbf{k}, t) \hat{F}_{i}^{\perp}\left(\mathbf{x}^{\prime}, \mathbf{k}^{\prime}, t^{\prime}\right)\right\rangle \\
& \quad \propto U(\mathbf{x}, t) f^{2}\left(\left|\mathbf{x}^{\prime}-\mathbf{x}\right| / 2\right) \mathrm{FT}\left(F_{i}^{\perp}\right)\left(\mathbf{k}+\mathbf{k}^{\prime}\right) e^{i \mathbf{k} \cdot \mathbf{x}+i \mathbf{k}^{\prime} \cdot \mathbf{x}^{\prime}} \delta\left(t-t^{\prime}\right),
\end{aligned}
$$

where FT means Fourier transform. We now show that our assumptions allow the small scales and the subfilter stresses to be expressed in terms of the mean velocity profile.

\section{Subfilter-scale solution}

The integration of (16) with respect to time is straightforward in the plane parallel case, and gives

$$
\begin{aligned}
& x=U(z)\left(t-t_{0}\right)+x_{0}, \quad y=y_{0}, \quad z=x_{z 0}, \\
& k_{x}=k_{x 0}, \quad k_{y}=k_{y 0}, \quad k_{z}=k_{z 0}-k_{x} \partial_{z} U\left(t-t_{0}\right),
\end{aligned}
$$

where $x_{0}, y_{0}, z_{0}, k_{x 0}, k_{y 0}$, and $k_{z 0}$ are the initial conditions of the ray. This equation shows that the parameter $R$ $=k_{z} / k_{x}$ can be used instead of $t$ to label the trajectories. To further simplify the notation we introduce

$$
\begin{aligned}
& k_{h}=\sqrt{k_{x}^{2}+k_{y}^{2}}, \\
& \theta=\arctan \left(k_{y} / k_{x}\right) .
\end{aligned}
$$

Note that $k_{h}$ and $\theta$ are constant along the rays. With this notation the subfilter-scale equations (15) become

$\begin{aligned} D_{R} \hat{u}= & -\left[\frac{2 \cos \theta}{1+R^{2}}-\frac{1}{\cos \theta}\right] \hat{w}+\frac{\nu k_{h}^{2}}{\cos \theta \partial_{z} U}\left(1+R^{2}\right) \hat{u} \\ & -\frac{1}{\cos \theta \partial_{z} U} \hat{F}_{u}^{\perp}, \\ D_{R} \hat{v}= & -\frac{2 \sin \theta}{1+R^{2}} \hat{w}+\frac{\nu k_{h}^{2}}{\cos \theta \partial_{z} U}\left(1+R^{2}\right) \hat{v}-\frac{1}{\cos \theta \partial_{z} U} \hat{F}_{v}^{\perp},\end{aligned}$

$D_{R} \hat{w}=-\frac{2 R}{1+R^{2}} \hat{w}+\frac{\nu k_{h}^{2}}{\cos \theta \partial_{z} U}\left(1+R^{2}\right) \hat{w}-\frac{1}{\cos \theta \partial_{z} U} \hat{F}_{w}^{\perp}$.

Note that the two-dimensional case can be found easily by putting $\theta=0$. Note also that when the forces are symmetric with respect to $y \rightarrow-y$, their Gabor transform is also symmetric with respect to $k_{y} \rightarrow-k_{y}$, i.e., $\theta \rightarrow-\theta$.

Since the forces are external, we can find the subfilterscale motions as the solution of a coupled linear inhomogeneous system of ordinary differential equations in $R$. In Appendix E, we show that this can be written as

$$
(\hat{u}, \hat{v}, \hat{w})(R)=\int_{-\infty}^{R}(\tilde{u}, \tilde{v}, \tilde{w})\left(R, R_{0}\right) d R_{0},
$$

where $(\tilde{u}, \tilde{v}, \tilde{w})\left(R, R_{0}\right)$ are solutions of the homogeneous part of (22) (i.e., with no force term), such that at $R=R_{0}$,

$$
(\tilde{u}, \tilde{v}, \tilde{w})\left(R_{0}, R_{0}\right)=-\frac{1}{\cos \theta \partial_{z} U}\left(F_{u}^{\perp}, F_{v}^{\perp}, F_{w}\right)\left(R_{0}\right) .
$$

This property can be used to compute formally the first subfilter term $\langle\overline{U w}\rangle$. Indeed, as shown in Appendix E, this term can be written 


$$
\begin{aligned}
\langle\overline{U w}\rangle= & \int_{-\pi}^{\pi} d \theta \int_{0}^{\infty} k_{h} d k_{h} \int_{-\infty}^{\infty} d R \int_{-\infty}^{R}\left\langle\hat{U}\left(\mathbf{x}, R, k_{h},-\theta\right)\right. \\
& \left.\times \tilde{w}\left(R, R_{0}, k_{h}, \theta\right)\right\rangle d R_{0} .
\end{aligned}
$$

Using the solutions of the homogeneous system given in Appendix E, and using the correlation between $U$ and $F(19)$, (25) becomes

$$
\begin{aligned}
\langle\overline{U w}\rangle= & -\frac{U}{\partial_{z} U} \int_{-\pi}^{\pi} d \theta \int_{0}^{\infty} k_{h} d k_{h} \int_{-\infty}^{\infty} d R \\
& \times \int_{-\infty}^{R} \mathrm{FT}\left(F_{w}^{\perp}\right)\left(\mathbf{k}+\mathbf{k}_{\mathbf{0}}\right) e^{i k_{x}\left(R+R_{0}\right) z} \\
& \times \frac{1+R_{0}^{2}}{1+R^{2}} \frac{\exp \left[-\frac{\nu k_{h}^{2} \int_{R_{0}}^{R}\left(1+X^{2}\right) d X}{\partial_{z} U \cos \theta}\right]}{\cos \theta} d R_{0} \\
\equiv & \frac{U}{\partial_{z} U} \eta(z) .
\end{aligned}
$$

Note that in the limit $\nu \rightarrow 0$, the integral diverges at $\theta$ $=\pi / 2$, but the viscosity regularizes this singular behavior. It is shown in Appendix $\mathrm{F}$ that in the limit where $\nu \rightarrow 0$, expression (26) can actually be expanded as

$$
\bar{w}=\frac{\eta_{0} \ln \left(\nu k_{*}^{2} / \partial_{z} U\right)+\eta_{1}}{\partial_{z} U}+O\left(\nu k_{*}^{2} / \partial_{z} U\right),
$$

where $k_{*}$ is a typical horizontal wave number and $\eta_{0}=0$ for isotropic forcing near $z=0$.

Similar results can be obtained for the other Reynolds stresses $\langle\overline{u w}\rangle$. One finds ${ }^{14}$ that the Reynolds stresses diverge in the limit $\nu \rightarrow 0$ and can be written as

$$
\langle\overline{u w}\rangle=\frac{\lambda}{\partial_{z} U},
$$

with

$$
\lambda=\frac{\lambda_{0} \partial_{z} U}{\nu k_{*}^{2}}+\lambda_{1} \frac{\ln \left(\nu k_{*}^{2} / \partial_{z} U\right)}{\partial_{z} U}+\lambda_{2}+O\left(\nu k_{*}^{2} / \partial_{z} U\right) .
$$

A detailed proof of this can be found in Ref. 14 and will not be repeated here. We can, however, make some remarks about the sign of $\lambda$ by using the following results: as shown in Appendix E, the averaged product $\langle\overline{u w}\rangle$ can also be written as a function of the product of solutions of the homogeneous part of (22) $\tilde{u} \tilde{w}$ as

$$
\begin{aligned}
\langle\overline{u w}\rangle= & -\int_{-\pi}^{\pi} d \theta \int_{0}^{\infty} d k_{h} \int_{-\infty}^{\infty} d R\left(\cos \theta \partial_{z} U\right) \\
& \times \int_{-\infty}^{R}\left\langle\tilde{u} \tilde{w}\left(R, R_{0}\right)\right\rangle d R_{0} .
\end{aligned}
$$

In the inviscid limit, straightforward manipulations of (22) produces the following relation between solutions of the homogeneous system:

$$
\begin{aligned}
\tilde{u} \tilde{w}= & \cos \theta\left(D_{R}\left[\frac{\tilde{u}^{2}-\tilde{v}^{2}+\tilde{w}^{2}}{2}\right]-\frac{\cos \theta}{\sin \theta} D_{R}[\tilde{u} \tilde{v}]\right. \\
& \left.-\frac{1}{\sin \theta} D_{R}[R \tilde{v} \tilde{w}]\right) .
\end{aligned}
$$

Inserting (31) in (30) and exchanging the order of integrals over $R$ and $R_{0}$, we find

$$
\begin{aligned}
\langle\overline{u w}\rangle & =\frac{\lambda}{\partial_{z} U}, \\
\lambda=- & \int_{-\pi}^{\pi} d \theta \int_{0}^{\infty} d k_{h} \int_{-\infty}^{\infty} d R_{0}\left(\cos \theta \partial_{z} U\right)^{2} \\
& \times\left[\frac{\tilde{u}^{2}-\tilde{v}^{2}+\tilde{w}^{2}}{2}\right]-\frac{\cos \theta}{\sin \theta}[\tilde{u} \tilde{v}]-\left.\frac{1}{\sin \theta}[R \tilde{v} \tilde{w}]\right|_{R_{0}} ^{\infty},
\end{aligned}
$$

where the notation $\left.a\right|_{R_{0}} ^{\infty}$ stands for $a(\infty)-a\left(R_{0}\right)$. Using the initial and asymptotic values of $\tilde{u}, \tilde{v}$ and $\tilde{w}$, one finds finally that

$$
\begin{aligned}
\lambda= & -\int_{-\pi}^{\pi} d \theta \int_{0}^{\infty} d k_{h} \int_{-\infty}^{\infty} d R_{0} \\
& \times\left[-\frac{F_{u}^{\perp} F_{w}^{\perp}\left(1+R_{0}^{2}\right) \arctan \left(1 / R_{0}\right)}{\cos \theta}\right. \\
& +\frac{\left|F_{w}^{\perp}\right|^{2}}{2}\left(1+R_{0}^{2}-2 R_{0}\left(1+R_{0}^{2}\right) \arctan \left(1 / R_{0}\right)\right. \\
& \left.\left.-\left(1+R_{0}^{2}\right)^{2} \arctan ^{2}\left(1 / R_{0}\right) \frac{\sin ^{2} \theta}{\cos ^{2} \theta}\right)\right] \\
= & \int_{-\pi}^{\pi} d \theta \int_{0}^{\infty} d k_{h} \int_{-\infty}^{\infty} d R_{0} \frac{3 F}{16 \pi k_{h}^{2}\left(1+R_{0}^{2}\right)} \\
& \times\left(1-\frac{\sin ^{2} \theta}{\cos ^{2} \theta} \arctan ^{2}\left(1 / R_{0}\right)\right) .
\end{aligned}
$$

Here, we have used (18) to simplify the expression. Expression (33), which is derived in the inviscid limit, shows interesting features. First, note that removing the angular integration and setting $\theta=0$, we obtain the expression of $\lambda$ in the two-dimensional limit. This expression only depends on $F$, and is clearly positive. This means that the "turbulent" Reynolds stress $-\langle\overline{u w}\rangle$ has the opposite sign of the viscous stress $\nu \partial_{z} U$. This result was previously obtained in Ref. 15 . It might be a consequence of the inverse cascade of energy arising in two-dimensional turbulence. In the threedimensional case, expression (33) is actually divergent, as noted in Ref. 14. Taking into account the fact that viscosity regularizes the Reynolds stress at $\theta=\pi / 2$, here we note that the divergence makes the expression of $\lambda$ negative. This means that for small enough $\nu$ (large Reynolds number), the turbulent stress can be expected to change sign and have the same sign as the viscous stress. This discussion is strictly only valid for isotropic forcing. 
Summarizing, we have found that the two Reynolds stresses appearing in our model can be expressed as (28) and (27). Here $\eta$, and $\lambda$ are functions of $z$ ( $\lambda$ is only via $\partial_{z} U$ ) and are the only parameters (functions) appearing in our model. They are not arbitrary free parameters, however, but are connected with the energy cascade from the large scales to the small scales. Of course, the amount of energy cascading depends upon the filter which is used, so these constants are filter-dependent. The exact values of $\eta$ and $\lambda$ can be obtained, in principle, from a numerical LES simulation where the contribution from the subfilter-scales, expressed by Eqs. (28) and (27) are computed self-consistently at each time step via the forces (8).

\section{Resolved scale solution}

Upon substitution of the Reynolds stresses by their expression, and neglecting the terms involving the viscosity, which are important only close to the surface (but see Sec. IV B for inclusion of these terms), we obtain the resolved scale equations,

$$
\partial_{z}\left(\frac{\eta\langle U\rangle}{\partial_{z}\langle U\rangle}+\frac{\lambda}{\partial_{z}\langle U\rangle}\right)=-u_{*}^{2} g_{3}(z)-\left\langle\sigma_{x}\right\rangle .
$$

We now nondimensionalize the length by $L=d$ (channel case) or $L=h$ (boundary layer case) and the velocities by $u_{*}$. Note that $\lambda$ is dimensionally equivalent to an energy transfer $\left(V^{3} / L\right)$. So we may write

$$
\lambda=\frac{u_{*}^{3}}{L} \tilde{\lambda},
$$

where $\tilde{\lambda}$ is a nondimensional constant. We stress again that $\tilde{\lambda}$ might be filter dependent, and that its exact value can be obtained via a LES simulation. For the term $\eta$, we note that the dimension of $\eta$ is $V^{2} / L$. So, we write it as

$$
\eta=\frac{u_{*}^{2}}{d} \tilde{\eta},
$$

where the refers to nondimensionalized quantities, such as $z / L$. With this nondimensionalization, and after one integration over $\tilde{z}$ of (34), we obtain the ordinary differential equation,

$$
\tilde{\eta} \frac{\widetilde{U}}{\partial_{\tilde{z}} \tilde{U}}+\tilde{\lambda} \frac{1}{\partial_{\tilde{z}} \widetilde{U}}=\int_{z_{0}}^{\tilde{z}}\left(\mathcal{S}(x)+\left\langle\sigma_{x}\right\rangle\right) d x .
$$

Here, we have introduced the location $z_{0}$ where the total (viscous plus turbulent) Reynolds stresses vanish (e.g., $z_{0}$ $=0$ for channel flows). The mean profile can then be obtained via integration of (37) as a function of $z$, for a given filter shape, once the value of $\tilde{\lambda}$ and $\tilde{\eta}$ is fixed. This shape can be found in principle from a numerical implementation of our subfilter model. Leaving this for future work, we prefer instead to examine the qualitative behavior of the parameters of our model, in order to construct qualitative analytical mean profiles.

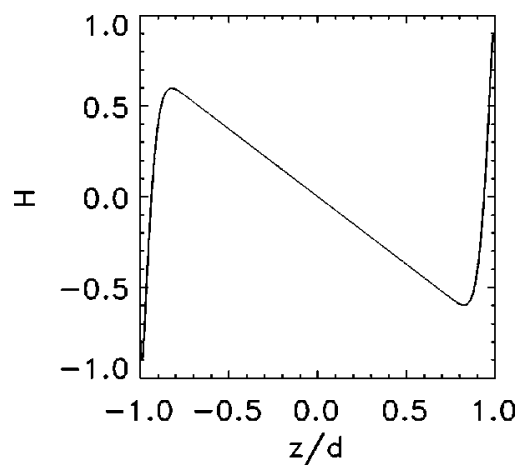

FIG. 1. Function $H$ defined by (38) (see appendices for details) for a channel with a Gaussian filter.

\section{E. Qualitative behavior of the model parameters}

Our model involves three parameter functions: $\tilde{\eta}, \tilde{\lambda}$ and

$$
H=\int_{z_{0}}^{\tilde{z}}\left(\widetilde{\mathcal{S}}\left(\tilde{z}^{\prime}\right)+\left\langle\sigma_{1}\right\rangle d \tilde{z}^{\prime}\right) \text {. }
$$

Their qualitative behavior can be found, for a given filter shape, via their definition and their high Reynolds number expansion. For example, it is convenient to use a Gaussian filter of width $l_{*}$, leading to $g_{3}(z)=N^{-1}\left(l_{*}\right)$ $\times \exp \left[-z^{2} /\left(2 l_{*}^{2}\right)\right]$, where $N\left(l_{*}\right)$ is a suitable normalizing quantity.

The computation of the function $H$ from its definition (38) is straightforward, and $H$ is shown in Figs. 1 and 2. Note the linear (channel case) or constant (boundary layer) behavior in the core or bulk region, and the deviations from the linear law near the boundaries. To examine the qualitative behavior of $\tilde{\eta}$ we use the expansion (27) and consider two interesting limits. In the near-wall limit, $\partial_{\tilde{z}} \widetilde{U} \rightarrow u_{*} d / \nu$, and so the contribution proportional to $\eta_{0}$ (logarithmic in $\partial_{z} U$ ) tends to zero. Then, to leading order in the viscosity, $\tilde{\eta}$ tends to a constant. In the core region, the forcing is isotropic, and $\eta_{0}$ tends to zero. Again, $\tilde{\eta}$ is approximatively constant with only a weak Reynolds number dependence. It is reasonable to assume that $\tilde{\eta}$ is piecewise constant (one constant near the wall, matched to another constant in the core).

For $\tilde{\lambda}$, we use expansion (27) and arguments similar to those used above: near the wall, we find that $\tilde{\lambda}$ tends to a

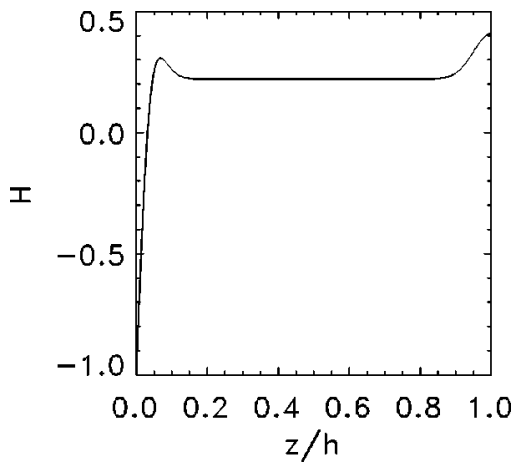

FIG. 2. Function $H$ defined by (38) (see appendices for details) for a boundary layer with a Gaussian filter. 
constant, with correction in the form of a power series in $\partial_{z} \widetilde{U}-R_{+}$. Since the turbulent Reynolds stresses at the wall must be zero, the constant is necessarily zero, and the expansion of $\tilde{\lambda}$ starts with a term linear in $\left(\partial_{z} U-R_{+}\right)$. This property is used in Sec. IV B. In the core, the forcing is isotropic, and $\lambda_{1}$ vanishes, giving no logarithmic correction in $\partial_{z} U$ to $\lambda$. Since $\partial_{z} \widetilde{U} \rightarrow 0$ in the core, the main behavior of $\tilde{\lambda}$ is then constant, with corrections proportional to $\left(\partial_{z} U\right)^{2}$; see Sec. IV A. Because of the isotropy in the core region, this constant is expected to be negative.

\section{MEAN VELOCITY PROFILE IN PLANE PARALLEL GEOMETRY}

\section{A. Velocity defect laws in the core region}

Equation (37) is valid far from the wall throughout the core region. To integrate it more easily, we introduce the path variable

$$
s=-\int_{0}^{\tilde{z}} \frac{\tilde{\eta}\left(z^{\prime}\right) d z^{\prime}}{\int_{z_{0}}^{z^{\prime}}\left(\mathcal{S}(x)+\left\langle\sigma_{x}\right\rangle\right) d x} .
$$

In terms of this path variable (37) becomes simply

$$
\frac{d \widetilde{U}}{d s}=\left(U_{*}-U\right)
$$

where $U_{*}=-\tilde{\lambda} / \tilde{\eta}$. Since $\tilde{\eta}$ and $\tilde{\lambda}$ are a priori both functions of $s, U_{*}$ is also a function of $s$. In the core region $U_{*}$ is approximatively constant $U_{*}(s)=U_{c}$ and (40) can be integrated to give

$$
U_{c}-U=\left(U_{c}-U_{D}\right) e^{s-s_{D}},
$$

where $s_{D}$ is the value of the path variable at the edge of the core region (where our approximations fail), and $U_{D}$ the value of the velocity at this location. Equation (41) is a velocity defect law, where the universal scaling function depends on the path variable, i.e., on the forcing and surface terms. Since these terms depend on the flow geometry, we can expect different velocity defect laws depending on the particular geometry of the flow.

\section{Channel flows}

In the case of channel flows, the surface terms are concentrated near the wall, and there is a constant streamwise pressure gradient. The typical behavior of $H=d z / d s$ and $s$ is shown schematically in Fig. 1 near the channel center. Near the center of the channel the surface terms vanish, and for constant or slowly varying $\tilde{\eta}$ the function $H$ is linear in $\tilde{z}$, which produces a logarithmic dependence of the path variable on $\tilde{z}$. Closer to the wall, the surface terms play a larger role, and small deviations from the logarithmic behavior for $s$ appear. These deviations are not universal, and depend weakly on the filter shape.

With a logarithmically varying path variable (41) leads to an algebraic defect law for the mean velocity,

$$
U_{c}-U=\left(U_{c}-U_{D}\right)\left|\frac{z}{z_{D}}\right|^{\gamma},
$$

where $\gamma=-\tilde{\eta} /\left\langle\sigma_{x}\right\rangle$. Oberlack $^{9}$ showed that this type of law is a consequence of a basic symmetry of the Navier-Stokes equations. It corresponds to the solution with the highest degree of symmetry. As such, he argued that the validity of this type of law is more likely to be confined to the channel center (as we have found where) than in the near-wall region, as claimed by Barenblatt. ${ }^{4}$ This assertion was supported by a careful analysis of various high and low Reynolds number numerical and experimental data. ${ }^{9}$ At high Reynolds number (between 18000 and 40000 ), Oberlack found $\gamma=1.69, z_{D}$ $=0.8 d$, and $U_{c}-U_{D}=4 u_{*}$. At lower Reynolds number, the exponent of the power law appeared to increase, suggesting that $\gamma$ depends weakly on Reynolds number. In terms of our model this is not surprising, since $\gamma$ is proportional to $\tilde{\eta}$, i.e., inversely proportional to $U_{c}$, which increases slowly with Reynolds number. The form of the dependence on Reynolds number can be derived using similarity arguments. ${ }^{19}$ One finds that $U_{c}=(1 / K) \ln R_{+}-U_{c 0}$, where $K$ is the von Karman constant, $R_{+}=u_{*} d / \nu$ is the Reynolds number based on the wall shear velocity and $U_{c 0}$ is a constant which may depend on the geometry. If this law is valid, and if $\lambda$ does not depend on the Reynolds number, then our model predicts that the exponent of the algebraic law should decrease like

$$
\gamma=\frac{\gamma_{0}}{\ln \left(R_{+} / R_{+0}\right)} .
$$

This is exactly the dependence assumed by Barenblatt ${ }^{4}$ and which leads, in the infinite Reynolds number limit, to the log defect law of the wall. Note, however, that we predict this behavior for the velocity defect law in the core region, using the outer variable $z$, while Barenblatt assumed this law in the near wall region, using the inner variable $z_{+}=R_{+}(1$ $+z / d)$. A recent analysis of Super Pipe data by Zagarola et $a l^{6,11}$ seems to contradict the theory by Barenblatt, while the study of Oberlack ${ }^{9,12}$ seems to confirm the existence of the algebraic defect law, even in the pipe geometry.

\section{Boundary layer}

In the boundary layer, there is no pressure gradient, and surface terms are important both near the surface and at the top of the boundary layer. The typical behavior of $H$ $=d z / d s$ and $s$ can then be shown by Fig. 2; in the core of the boundary layer the surface terms vanish, and $\tilde{\eta}$ and the function $H$ are constant, resulting in a linear dependence of the path variable on $\tilde{z}$. Near the surface and at the top of the boundary layer the surface terms become more important, and small deviations from the linear behavior for $s$ appear. As in the case of the channel, the deviations are not universal and depend weakly on the filter shape.

With a linearly varying path variable, Eq. (41) leads to an exponential defect law for the mean velocity,

$$
U_{\infty}-U=\left(U_{\infty}-U_{D}\right) e^{-\beta z / z_{D}},
$$

where $\beta=-\tilde{\eta} / H(h / 2)$. This type of law is also a consequence of a basic symmetry of the Navier-Stokes equations. ${ }^{9}$ The experimental validation of this law is difficult, as discussed by Oberlack. ${ }^{9}$ To check it one needs to 
subtract the free velocity $U_{\infty}$. Therefore, a few percent error in this quantity results in a large error in the determination of the coefficients, and a bad determination of the universal curve in log-coordinates. Oberlack nevertheless obtained a very good agreement with experimental data for $\beta=9.46$, $z_{D}=\Delta_{c}$, the Rotta-Clausius length, and $U_{\infty}-U_{D}$ $=10.34 u_{*}$.

To our knowledge, our model is the first dynamical derivation of this exponential defect law predicted by Oberlack on symmetry arguments. Our model also explains why this type of law is observed in the boundary layer, rather than in channel flows; this appears to be linked to the absence of pressure gradients.

\section{Plane Couette flow}

Plane Couette flow represents an interesting special case: because of the antisymmetry of the problem with respect to $z \rightarrow-z$, the mean flow is necessarily zero at the center. This has some important consequences on energy production and transfers in the core: in a shear flow, energy production and nonlinear transfers occur mainly as a result of interaction with the mean pressure gradient, or nonlocal nonlinear interactions with the mean flow. We thus expect the "forcing" (nonlinear energy transfer) on the small-scales to be zero at the core, i.e., $\tilde{\eta}$ should be zero at the center of the channel. With zero $\tilde{\eta}$, we may redefine the path variable as

$$
s=-\int_{0}^{\tilde{z}} \frac{\tilde{\lambda} d z^{\prime}}{\int_{z_{0}}^{z^{\prime}}\left(\mathcal{S}(x)+\left\langle\sigma_{x}\right\rangle\right) d x} .
$$

Since there are no pressure gradients, this path variable is linear in $z$, as in the boundary layer case. The integration of (37) then leads to

$$
U=\beta \tilde{z},
$$

where $\beta=\lambda / H(0)$. This linear law at the center is also a special case of symmetric solution of Navier-Stokes equations. ${ }^{9}$ In fact, it corresponds to the solution with the lowest degree of symmetry. Investigation of experimental or numerical data led Oberlack to conclude that this linear law was valid to very high accuracy over about $80 \%$ of the core region. ${ }^{9}$ It is remarkable that our model also leads to this linear case as a special outcome of the (anti)-symmetry of the mean flow (Table I).

\section{B. Near-wall region and universal log-laws}

We just saw that our model leads to general predictions about mean equilibrium profiles in the core region which are in remarkable agreement with those obtained using symmetry by Oberlack. ${ }^{9}$ It is thus natural to investigate the kinds of predictions we can get from our model in the near-wall region. For this, we need the behavior of our subfilter tensors near the wall. We use (27) and (29) to perform simple Taylor expansions in the near-wall region, as a function of $\partial_{z} \widetilde{U}$. Specifically, we consider first the function $\tilde{\lambda}$. For our subfilter tensors to be zero at the wall, as required by boundary
TABLE I. Parameter used for the expression of the fitted functions $H(z)$ and $\eta(z)$.

\begin{tabular}{clll}
\hline \hline Cases & Superpipe & \multicolumn{1}{c}{ Channel } & Boundary layer \\
\hline$z_{w}$ & 0 & -1 & 0 \\
$z_{c}$ & $R_{*}$ & $z_{t}$ & $z_{t}$ \\
$d H_{w}$ & $\frac{K R_{+}}{1+K R_{*}}$ & $\frac{K R_{+}}{1+K R_{*}}$ & $\frac{K R_{+}}{1+K R_{*}}$ \\
$d H_{c}$ & - & $-\frac{K R_{*}}{1+K R_{*}}\left(1+\frac{R_{+}\left(1+z_{t}\right)}{R_{*}}\right)$ & 0 \\
$\eta_{w}$ & $\eta_{0} \frac{K R_{+}}{1+K R_{*}}$ & 0 & 0 \\
$\eta_{c}$ & 0 & $\alpha d H_{c}$ & $\frac{1}{U_{\infty}} \frac{R_{+}}{K R_{*}}$ \\
\hline
\end{tabular}

conditions, we must have $\tilde{\lambda}+\tilde{\eta} \widetilde{U}=0$ at the wall. This is guaranteed if and only if $\tilde{\lambda}$ is itself zero at the wall. We may then expand it near the wall as

$$
\tilde{\lambda}=\lambda_{0}\left(\partial_{z} U-R_{+}\right)+\lambda_{1}\left(\partial_{z} U-R_{+}\right)^{2}+\cdots, \partial_{z} U \rightarrow R_{+},
$$

where $\lambda_{0}$ and $\lambda_{1}$ are some constants, and the Reynolds number $R_{+}=u_{*} L / \nu$ is the nondimensional value of the viscous flux at the wall. We have kept the largest relevant order, as we shall see later. For $\tilde{\eta}$, we use the qualitative behavior discussed in Sec. III E and take it to be a constant (which can be zero).

To obtain the equilibrium equation in the near-wall region, we then substituted this development into the original Eq. (14). After series expansions and rearrangements, these equations become [after a change of variable from outer to inner variables $\left.\tilde{z} \rightarrow z_{+}=R_{+}(1+\tilde{z})\right]$

$$
\frac{1+K R_{*} \tilde{\eta} \widetilde{U}}{\dot{U}}=K R_{*}\left(\frac{z_{+}}{R_{*}}-1\right)+\left(1+K R_{*}\right) \dot{U}
$$

where the dot refers to a derivative with respect to $z_{+}$and we have introduced

$$
\begin{aligned}
& K=\frac{1}{R_{+}\left(1-\lambda_{1} R_{+}\right)}, \\
& R_{*}=R_{+}\left(1+\lambda_{0}-2 \lambda_{1} R_{+}\right) .
\end{aligned}
$$

Equation (47) encompasses two kinds of variation: very close to the wall, for $z_{+} \ll\left|R_{*}\right|$, where $\lambda \approx \lambda_{0}$ and $U / R_{+}$ $\ll 1$, we have the usual viscous behavior $\partial_{z_{+}} \widetilde{U}=1, \widetilde{U}=z_{+}$. This suggests that $\left|R_{*}\right|$ should be interpreted as the size of the viscous sublayer, which is usually observed to be of the order of 10 wall inner units. Further away from the wall, for $\left|R_{*}\right| \ll z_{+}<z_{0}$, the equation can be simplified to

$$
\frac{1+K R_{*} \tilde{\eta} \widetilde{U}}{\dot{U}}=K z_{+} .
$$


This equation has two types of solutions for slowly varying $K, R_{*}$ and $\tilde{\eta}$ : when $\tilde{\eta}$ is different from zero, the solution is algebraic in the inner variable,

$$
\begin{aligned}
& U-\frac{1}{K R_{*} \tilde{\eta}}=\left(U_{0}-\frac{1}{K R_{*} \tilde{\eta}}\right)\left(\frac{z_{+}}{z_{0}}\right)^{\alpha}, \\
& \alpha=\tilde{\eta} R_{*} .
\end{aligned}
$$

This solution is the generic near-wall solution in the present model. It would be exactly the solution proposed by Barenblatt ${ }^{4}$ if we allowed the (unknown) parameter $\alpha$ to decrease with the Reynolds number like $\alpha \sim 1 / \ln \left(R_{+} / R_{0}\right)$. At the present time, we have no means to checking this possibility. We note, however that Zagarola et al.' $\mathrm{s}^{6}$ analysis of the Super Pipe data indicates the existence of a power law regime for $50<z_{+}<500$ which would correspond to $\tilde{\lambda}=0$ and $\alpha=0.137$. In this near-wall region, curvature effects are very small, and the pipe geometry is equivalent to a plane parallel geometry, so we may interpret this finding as a confirmation of our generic solution.

When $\tilde{\eta}=0$, we obtain the celebrated log law of the wall

$$
\widetilde{U}=\frac{1}{K} \ln z_{+}+B,
$$

which shows that $K$ may be interpreted as the von Karman constant. Note that we can obtain an exact expression of $B$ in the case where $R_{*}$ and $K$ are constants, independent of $z_{+}$. Indeed, when $\tilde{\eta}=0$, there is an exact analytical solution of (47)

$$
\begin{aligned}
& \Delta\left(z_{+}\right)=\sqrt{\left(1-z_{+} / R_{*}\right)^{2}+4 \frac{1+K R_{*}}{\left(K R_{*}\right)^{2}}}, \\
& \dot{U}=\frac{K R_{*}}{2\left(1+K R_{*}\right)}\left(1-\frac{z_{+}}{R_{*}}+\Delta\right), \\
& U=-\frac{K R_{*}^{2}}{4\left(1+K R_{*}\right)}\left[\left(1-z_{+} / R_{*}\right)^{2}\right. \\
& \left.\quad+\left(1-z_{+} / R_{*}\right) \Delta\left(z_{+}\right)\right]-\frac{1}{K} \ln \left(2 \Delta\left(z_{+}\right)\right. \\
& \left.\quad+2\left(1-z_{+} / R_{*}\right)\right)+\frac{R_{*}}{2}+\frac{1}{K} \ln \frac{4\left(1+K R_{*}\right)}{K R_{*}} .
\end{aligned}
$$

Note that (53) is the generic two parameter formula describing the whole near wall behavior, from the viscous sublayer to the logarithmic region. This formula is a direct consequence of our subfilter model, and could be used, for example, to better estimate the friction velocity at the wall.

For $z_{+} \gg R_{*}$, this solution reduces to

$$
\begin{aligned}
& U=\frac{1}{K} \ln z_{+}+B, \\
& B=\frac{1}{2 K}\left(1+K R_{*}+2 \ln (K)\right) .
\end{aligned}
$$

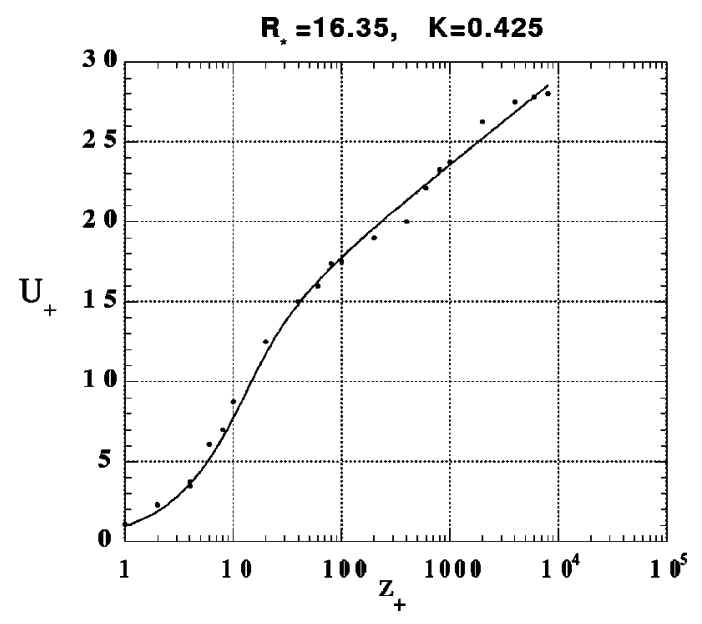

FIG. 3. Velocity profiles: our model (line), data of Kestin and Richardson (Ref. 25) (symbol).

This fixes the value of $B$ as a function of $K$ and $R_{*}$. The general formula agrees very well with some old boundary layer data. ${ }^{25}$ In Fig. 3, we show a comparison of formula (53) with the data of Kestin and Richardson. ${ }^{25}$ This best fit is obtained with $R_{*}=16.35$ and $K=0.425$, resulting in $B$ $=7.34$. We also tried to fit our general formula with the most recent Super Pipe data. The best fit, obtained with $R_{*}$ $=14.5$ and $K=0.45$, leading to $B=6.59$ (independent of Reynolds number) is shown in Fig. 4, where the solution (53) is compared with the data taken at 26 different Reynolds numbers spanning three decades. We resolve the transition between the viscous sublayer to the log-layer very well, and this fit captures the universal log-law observed at the different Reynolds numbers. However, in the transition zone between the viscous layer and the log-region, there is an additional bump in the data which is not captured by the model. Re-examining the data analysis by Zagarola et al., we see that this bump is possibly associated with an algebraic intermediate region. Such algebraic behavior can be obtained within our model only if $\tilde{\eta}$ is different from zero. On the other hand, the existence of the log-law for $z_{+}$larger than a

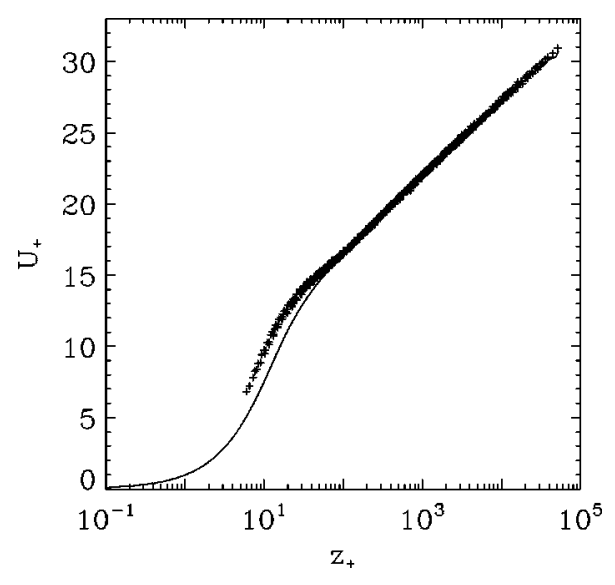

FIG. 4. Velocity profiles obtained with our model (line) $\left(R_{*}=14.5, K\right.$ $=0.45$, and $\eta=0$ ) compared to the data of Zagarola (Ref. 11) (symbols): results for 26 values of $R_{+}$between $3.15 E+04$ and $3.52 E+07$. 


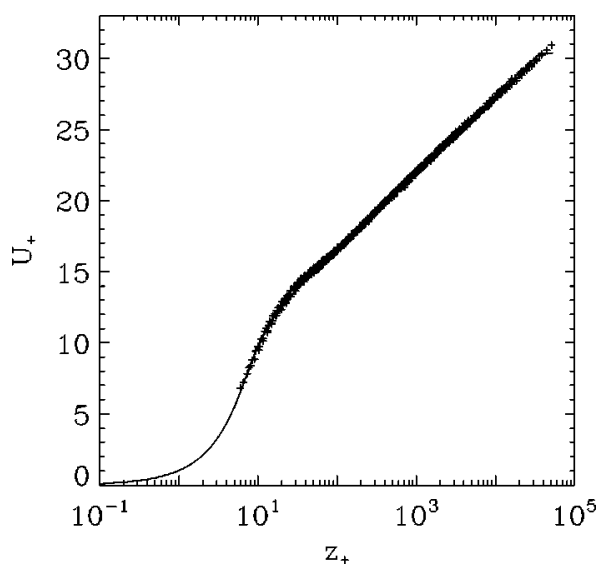

FIG. 5. Velocity profiles obtained with our model (line) $\left(R_{*}=6.8, K\right.$ $=0.45$, and $\eta=0.185$ ) compared to the data of Zagarola (Ref. 11) (symbols): results for 26 values of $R_{+}$between $3.15 E+04$ and $3.52 E+07$.

few $R_{*}$ precludes the possibility that $\tilde{\eta}$ is different from zero for $z_{+} \gg R_{*}$. We then explored a simple refinement of our procedure, in which $\tilde{\eta}$ is taken as a constant over $0<z_{+}$ $<R_{*}$, and in which, for universality reasons, this constant is proportional to the Reynolds number $\tilde{\eta}=\eta_{0} R_{+}$(Fig. 6). Tuning the three constants $K, R_{*}$ and $\eta_{0}$ in this new model, we get the best fit as shown in Fig. 5 with $K=0.45, R_{*}$ $=6.8$, and $\eta_{0}=0.185$. The fit is excellent over the whole spatial range, and over four decades of Reynolds numbers which, for a three parameter model, is encouraging.

\section{Models for the whole flow}

To push our model to the limit, we now use it to develop a single model for the entire flow region, from the viscous layer to the core region or the top of the boundary layer (depending on the flow geometry). For this, we employ matched asymptotic expansions between the core region (Sec. IV A) and the near-wall region (Sec. IV B). The idea is to use the asymptotic constraints on $\tilde{\lambda}$ and $\tilde{\eta}$ at the wall and in the core, obtained through boundary conditions and the series (28) and (27). This results in a constant $\tilde{\eta}$ at the wall and in the core (the two constants can be different). The

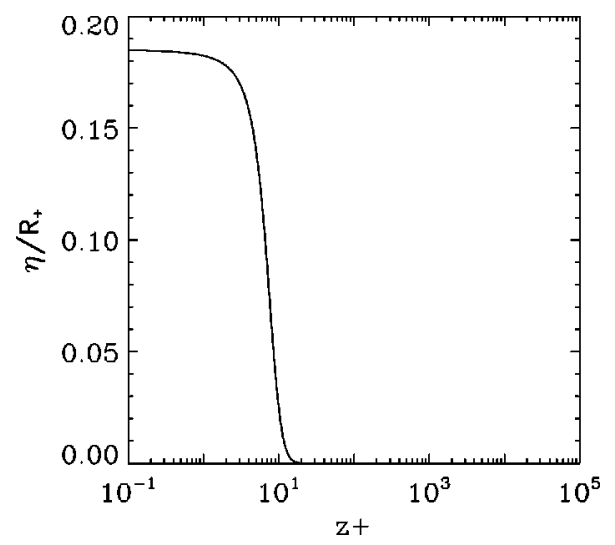

FIG. 6. Function $\eta\left(z_{+}\right)$used in our model to fit the data of Fig. 5 (see appendices for expression).
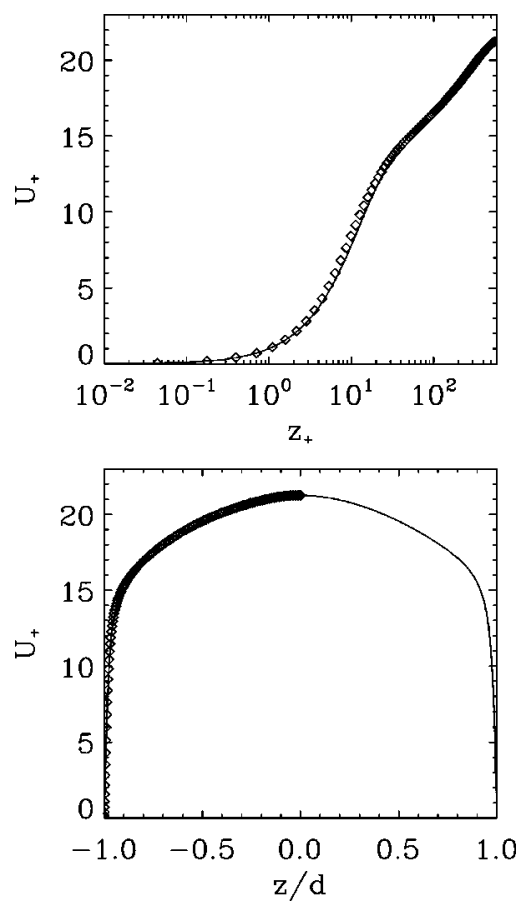

FIG. 7. Velocity profile at $\operatorname{Re}=587.19$ : Moser's data (Ref. 26) (symbol), our analytical law (line).

asymptotic constraints for $\tilde{\lambda}$ at the wall are given by (46). In the core, or at the top of the boundary layer, the constraints are obtained by the requirement of a finite subfilter tensor at the location $\partial_{z} U=0$ (the channel center, or the top of the boundary layer). This fixes the development of $\tilde{\lambda}$ as

$$
\tilde{\lambda}=-\tilde{\eta} U_{c}+\lambda_{z} \partial_{z} U-\nu_{t}\left(\partial_{z} U\right)^{2}+\cdots, \quad \partial_{z} U \rightarrow 0 .
$$

Here, $U_{c}$ is the velocity at the channel center or at the top of the boundary layer, and $\lambda_{z}$ and $\nu_{t}$ are unknown functions of $z$. The parameter $\nu_{t}$ is the equivalent of a nondimensional turbulent viscosity (see Sec. IV D). With this expansion, the total equation (14) becomes

$$
\frac{\tilde{\eta}\left(U-U_{c}\right)}{\left(R_{+}^{-1}+\nu_{t}\right) \partial_{z} U}=\frac{\left\langle\sigma_{x}\right\rangle \tilde{z}-\lambda_{z}}{R_{+}^{-1}+\nu_{t}}+\partial_{z} U .
$$

This equation is similar to the near-wall equation, except for the "forcing function" $H_{\text {core }}=\left(\left\langle\sigma_{x}\right) \tilde{z}-\lambda_{z}\right)\left(R_{+}^{-1}+\nu_{t}\right)$. This suggested to us the following procedure for fitting the whole flow: solve the equation

$$
\frac{\lambda_{\mathrm{eff}}+\tilde{\eta}_{\mathrm{eff}} U}{\partial_{z} U}=H_{\mathrm{eff}}+\partial_{z} U
$$

where $H_{\text {eff }}$ is a continuous function going from its near-wall behavior $H=-K R_{*} /\left(1+K R_{*}\right)$ to the core behavior $H_{\text {core }}$,

TABLE II. Parameter used for the fit of Moser's data.

\begin{tabular}{cccc}
\hline \hline Reynolds & $z_{t}$ & $R_{*}$ & $\alpha$ \\
\hline 178.82 & -0.800 & 19 & 1.04 \\
392.27 & -0.900 & 16 & 1.68 \\
587.19 & -0.924 & 14 & 1.85 \\
\hline \hline
\end{tabular}




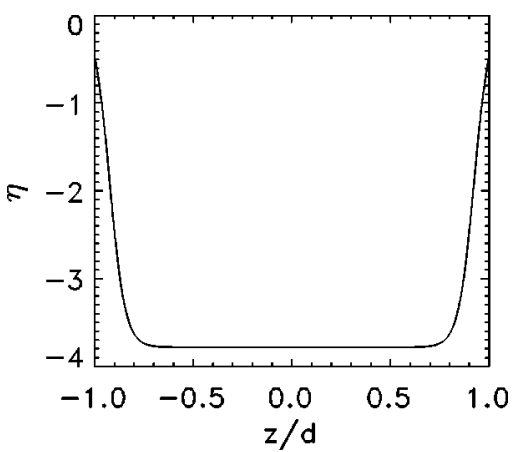

FIG. 8. Function $\tilde{\eta}_{\text {eff }}(\tilde{z})$ used to fit the data in Fig. 7 (see appendices for expression).

and $\lambda_{\text {eff }}$ and $\eta_{\text {eff }}$ are simple smooth functions going from their near wall value (see Sec. IV B), to their "core" value (Sec. IV A). We obtained the following results.

\section{Channel flows}

In this geometry, the integrated momentum flux must be zero at the center. This fixes $\lambda_{z}=0$. The simplest smooth function which extrapolates the near-wall behavior, and is linear in the core and zero at the center is shown in Fig. 6. We fixed the constant in the near-wall region so that the log-law is obtained with a von Karman constant of 0.45. We also fixed the velocity at the center $U_{c}$. We are then left with three free parameters: the location of the transition between near-wall and core behavior $z_{t}$ (see Fig. 7), the value of $\eta$ and the value of $R_{*}$, which governs the viscous/turbulent transition. We varied the three parameters and used a leastsquare procedure to choose the best value to fit the data. An example of a fit of the numerical data is shown in Fig. 7. We fitted the numerical data of Moser et al. ${ }^{26}$ which are available on the web at http://www.tam.uiuc.edu/ Faculty/Moser/Channel. The parameters for each of the three Reynolds number are listed in Table II. This fit was obtained with the function $H_{\text {eff }}$ and $\tilde{\eta}$ shown in Figs. 8 and 9. The corresponding turbulent momentum flux is shown in Fig. 10. It agrees very well with data from numerical simulations. $^{23}$

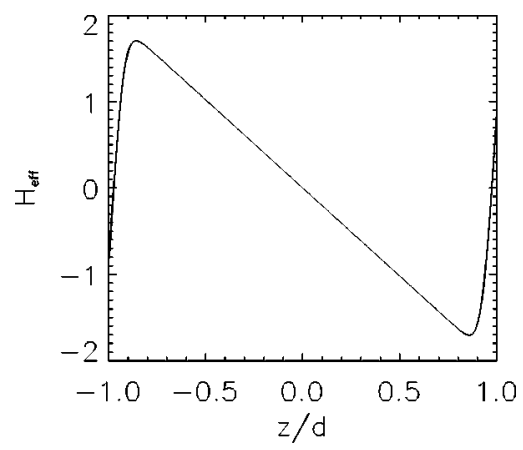

FIG. 9. Function $H_{\text {eff }}(\tilde{z})$ used to fit the data in Fig. 7.

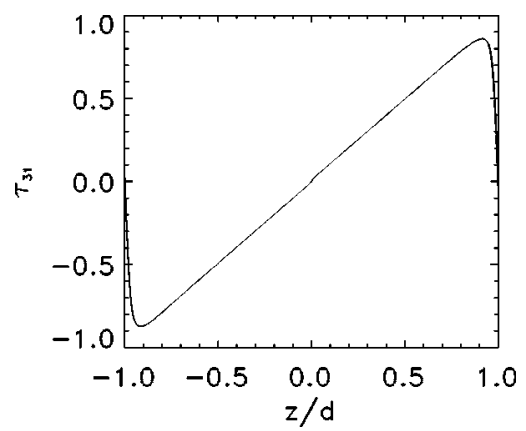

FIG. 10. Turbulent momentum flux $\tau_{31}$.

\section{Boundary layer}

In this geometry, there is no pressure gradient, but there can be a nonzero integrated momentum flux near the top of the boundary layer. The simplest smooth function extrapolating the near wall behavior and constant near the top of the boundary layer is shown in Fig. 13. We fixed the constant in the near-wall region so that the log-law is obtained with a von Karman constant of 0.44 . We also fixed the velocity at the top of the boundary layer to $U_{\infty}$. We are then left with three free parameters: the location of the transition between near-wall and core behavior $z_{t}$ (see Fig. 11), the value of $\eta$ and the value of $R_{*}$, which governs the viscous/turbulent transition. We varied the three parameters and used a leastsquare procedure to choose the best value to fit the data. An example of a fit to the numerical data of Nockemann et al. ${ }^{27}$ is shown in Fig. 11. This fit was obtained with the function $H_{\text {eff }}$ and $\tilde{\eta}$ shown in Figs. 12 and 13.
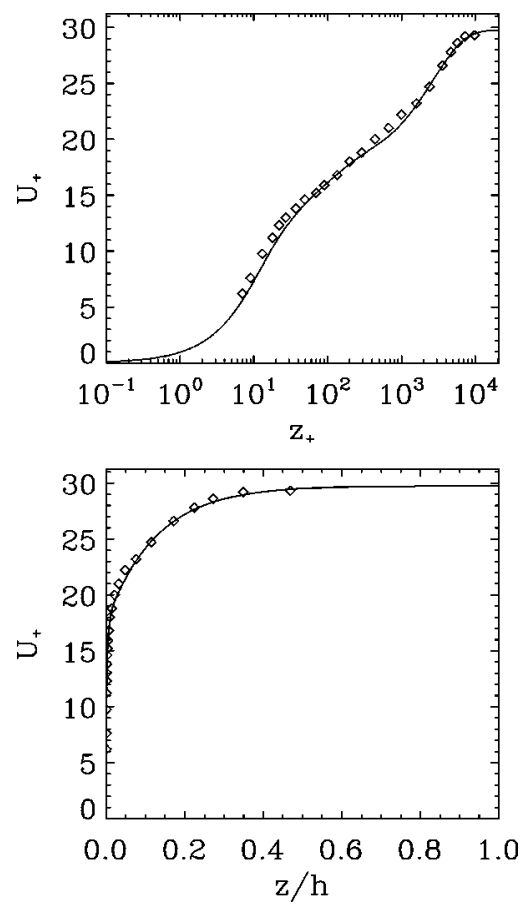

FIG. 11. Velocity profile at $\mathrm{Re}=20$ 920: Nockemann's data (Ref. 27) (symbol), our analytical law (line). 


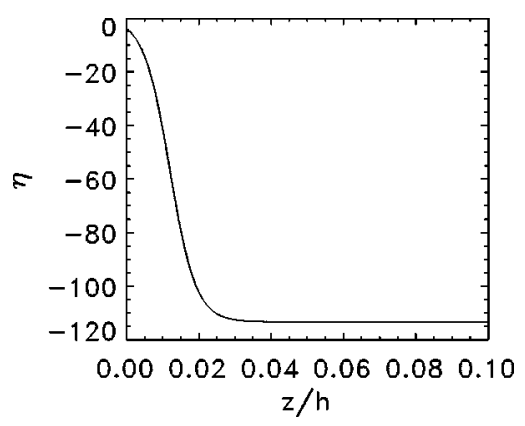

FIG. 12. Function $\tilde{\eta}_{\text {eff }}(\tilde{z})$ used to fit the data in Fig. 11 (see appendices for expression).

\section{Discussion}

Traditional stochastic averaged approaches for shear flows usually attempt to model the entire Reynolds stress $\tau_{13}=\langle(u+U)(w+W)\rangle$. In the present approach, however, we first use a spatial vertical filter to separate the velocity into its resolved and subfilter-scale parts, and then model each term separately via a nonlocal approximation. We obtained the final result,

$$
\tau_{13}=\frac{\lambda+\eta\langle U\rangle}{\partial_{z}\langle U\rangle},
$$

where $\lambda$ and $\eta$ are functions of $\partial_{z} U$ only and depend on the energy transfers from the resolved to the subfilter scales. In addition, their behavior is not known a priori, but we were able to obtain their near-wall and core behavior from asymptotic expansions, which provides a more quantitative picture of $\tau_{31}$. Using this expansion the Reynolds stress can be put in the form

$$
\tau_{31}=-\nu_{t *} \partial_{\tilde{z}}\langle U\rangle+\tau_{*}+\frac{\lambda_{0}+\eta_{0}\langle U\rangle}{\partial_{z}\langle U\rangle},
$$

where $\nu_{t *}, \tau_{*}, \lambda_{0}$, and $\eta_{0}$ are constants which are different in the near-wall and core regions. The first term in (59) is the standard eddy-viscosity. A striking consequence of this result is that in order to fit the whole turbulent channel flow data, one needs to impose a positive eddy viscosity at the wall, of the order of $\nu_{t}=K R_{*} u_{\tau}^{2} / \nu \approx 3 u_{\tau}^{2} / \nu$, and a negative eddy viscosity at the core, of the order of $\nu_{t}=-3 /\left(2 R_{+}\right)$. This last result is in good agreement with a previous theoret-

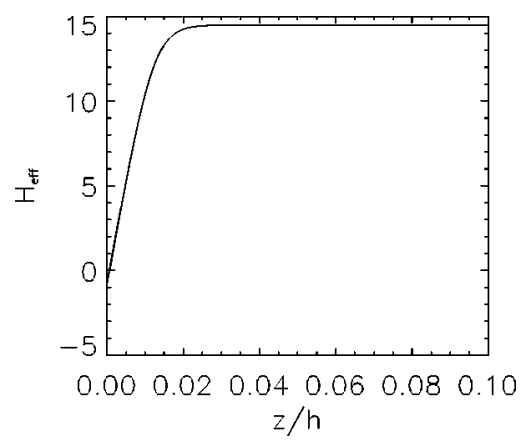

FIG. 13. Function $H_{\text {eff }}(\tilde{z})$ used to fit the data in Fig. 11 (see appendices for expression). ical asymptotic expansion for periodic shear flows (no boundary or wall regime), which showed that any eddy viscosity will necessarily become negative beyond a certain critical Reynolds number. ${ }^{28}$

Our analytical integration shows that our subfilter model naturally leads to all the different mean profiles which have been experimentally observed. Moreover, we have shown how boundary conditions and external pressure lead to the selection of a peculiar profile, and how a smooth matching of the "external" (at the boundaries) and "internal" (at the center) solution led to velocity profiles in very good agreement modern available data. The only uncertainty lies in the values of the numerical constants which appear in the analytical integration (e.g., the von Karman constant, or the value of $\eta$ near the wall), which we cannot predict analytically a priori since they are related to the energy cascade from large to small scales via the forcing (which we have prescribed). The ultimate test of our model will thus be a numerical implementation, which will produce numerical values of the constants which can then be compared with analytical data.

From a theoretical point of view, our analytical approach leads to a number of new results regarding shear flow dynamics and modeling. Our study first shows that a linear mechanism of rapid distortion of the small, turbulent scales, by the mean large-scale flow is responsible for the variety of shear profiles which are observed experimentally (log, linear, algebraic, and exponential). This result can be seen as the physical counterpart of the mathematical result obtained by Oberlack using a Lie group analysis of the Navier-Stokes equations. This result also complements the analysis of Farell and Ioannou ${ }^{29}$ who showed that in a shear flow the solutions of the linearized equations of motion, forced by a stochastic noise are the various coherent structures (streaks, hairpin vortices, ... ) identified in numerical simulations or experiments of shear flows. This supports the hypothesis that the small-scale structure is determined mainly by interaction with the mean flow.

From a practical point of view, our asymptotic expansion leads to an analytic expression for the transition between the viscous- and log-layer. This formulation depends on three parameters: the von Karman constant $K$, the size of the viscous layer $R_{*}$, and $\eta$ which characterizes the mean forcing acting on the subfilter velocities in the mean streamwise direction. The first two parameters are sufficient to describe the transition from the linear viscous profile near the wall, to the log-profile away from the wall. The third parameter $\eta$ is required to describe the "bump" observed in the high Reynolds number Super Pipe data. This parameter, however, does not correspond to any classical turbulent viscosity. It is therefore interesting to compare our formulation, with previous empirical models of the turbulent subfilterscale tensor. This is traditionally obtained via a turbulent viscosity model,

$$
\tau_{13}=\nu_{t} \partial_{z} U
$$

where $\nu_{t}$ is the turbulent viscosity. In order to reproduce accurately the profile variations near the wall, one is usually forced to take a turbulent viscosity that varies with $z$, such 
that it is zero at the wall. One popular model ${ }^{30}$ includes exponential damping of the viscosity at the wall, over some characteristic length scale $l_{*}=26 \nu / u_{*}$. In our case, we have taken into account the near-wall damping in our asymptotic expansion. It occurs over a length-scale $l_{*}=R_{*} \nu / u_{*}$ $\approx 7 \nu / u_{*}$. The shape of the damping in our case, can be found by comparing (60) with (59). Our description corresponds to an "effective" turbulent viscosity,

$$
\nu_{t}^{\mathrm{eff}}=\nu_{t *}+\frac{\tau_{*}}{\partial_{z}\langle U\rangle}+\frac{\lambda_{0}+\eta_{0}\langle U\rangle}{\left(\partial_{z}\langle U\rangle\right)^{2}} .
$$

Thus, in our model the damping occurs self-consistently via the velocity gradients.

It is also interesting to interpret the "turbulent" lengthscale implied by our model. This length-scale appears when the turbulent viscosity is expressed as a function of the mean shear $\partial_{z}\langle U\rangle$,

$$
\nu_{t}=C l^{2} \partial_{z}\langle U\rangle
$$

Comparison of our model with (62) shows that the two models are compatible provided the turbulent length-scale varies like

$$
l \sim \frac{\sqrt{\tilde{\lambda}+\tilde{\eta} U}}{\partial_{z}\langle U\rangle} .
$$

Such a shape has so far never been proposed in a turbulent model based on dimensional arguments. Its is therefore useful to compare it with standard empirical models. Near the wall, the velocity profile is logarithmic and the mean velocity is small so the corresponding length-scale varies linearly with the distance from the wall. This is in agreement with the standard single length-scale description of turbulent flows near the wall. Farther from the wall the change in lengthscale depends on the geometry. For channel flows, one can actually show that the mean velocity profile in the core region varies algebraically (see Sec. IV A) with the distance from the core, and one can expect a weak variation of the length-scale in the bulk of the flow, followed by a divergence at the center of the channel. This divergence may explain the scatter in measurements of the turbulent length-scale at that location. Note that the inverse dependence of the lengthscale (63) on the mean gradient is similar to the empirical description of the length-scale proposed by Hunt et al., ${ }^{31}$

$$
\frac{1}{l}=\frac{A}{z_{+}}+B \frac{\partial_{z} U}{\sqrt{\left\langle w^{2}\right\rangle}}
$$

where $z_{+}$is the distance from the wall, and $A$ and $B$ are arbitrary parameters. This description gives the same qualitative behavior as ours; near the wall the behavior is dominated by the first term (giving the usual linear law for the length scale), while far from the wall the second term dominates (giving a linear dependence of $1 / l$ with the mean velocity gradient). This is precisely the dependence predicted by our model.

\section{SUMMARY}

In this paper we have developed a new model for plane parallel flows which involves the coupling of two dynamical equations: one for the resolved scales (which depends upon the Reynolds stresses generated by the subfilter motions), and one for the subfilter-scales (which can be used to compute the subfilter-scale Reynolds stresses). The subfilterscales evolve according to a linear inhomogeneous equation, which is forced by the energy cascade from resolved to subfilter-scales. Under the assumption of $\delta$-correlations of the forces the subfilter-scale equation can be formally integrated, which leads to an expression for the subfilter-scales as a function of the resolved motions only.

We then used this analytical expression for the subfilterscales to study the possible equilibrium profiles arising in a plane geometry. In the core region our classification of the equilibrium profiles agrees with the system derived by Oberlack $^{12}$ from the basic symmetries of the Navier-Stokes equations. This shows that our model respects all the basic symmetries of the Navier-Stokes equations. In the near-wall region we used an asymptotic expansion to obtain a theoretical description of the complete transition region between the viscous layer and the log-region. We showed that an excellent fit to the recent data from the Super Pipe experiments can be found from our theory, which includes a new quantity describing the effect of the energy cascade from resolved to subfilter-scales. Finally, we showed that our formulation gives a description of the equilibrium profiles across the whole turbulent channel via a simple matching procedure.

From a theoretical point of view, it is interesting to compare the results of our approach with the recent similarity theories of Barenblatt ${ }^{4}$ and George et al. ${ }^{7,8}$ In the near-wall region, we find that the relevant scaling variables are the friction velocity and the inner length scale $z_{+}$, in agreement with George. We also predict two different possible nearwall velocity profiles, the algebraic profile of Barenblatt and George, or the more traditional logarithmic profile. The selection of one or the other depends on the properties of the forcing due to the energy cascade from large to small scales (see also Ref. 14), which we cannot study analytically. On the other hand, we have no clear indication that the exponent of the possible power law is Reynolds number dependent (as predicted by Barenblatt and George), nor do we obtain the shifted logarithmic law predicted by George [our general formula is given in (53) and is more complicated than a shifted log-law]. In the core region, we find that the relevant scaling variables are the central velocity and the outer length scale $z / d$, in agreement with George. We obtain various different velocity defect laws according to the geometry; in channel flows, we predict the occurrence of algebraic (or logarithmic, for very special energy cascades) velocity profiles, in agreement with George. For zero-pressure boundary layer, however, our prediction is an exponential velocity profile, in contradiction with the algebraic prediction of George. Note that exponential laws might sometimes be confused with algebraic laws with very large exponent in a data analysis involving large error bars. This is typically the case of boundary layer experiments, since the determination of the velocity 
defect law implies the very precise knowledge of the asymptotic velocity, which is not always available.

Together, these results confirm the interest of our new subfilter-scale approach, which uses an approximation to subfilter-scale velocities to compute the subfilter-scale Reynolds stresses (and hence equilibrium flow profiles). We are now attempting to numerically integrate our set of coupled equation in order to find completely quantitative solutions to the problem of plane parallel flows at high Reynolds number.

From a more general perspective, it is interesting to note that the basic approximation use to derive our subfilter model from the Navier-Stokes equation (the nonlocality of subfilter energy transfers) could be easily applied to other type of flows (including, e.g., temperature, magnetic fields, rotation) and geometry to derive equivalent subfilter models in a systematic way (see, e.g., Refs. 33-35). The quality of the corresponding model would of course depend on the validity of the nonlocality approximation in the given flow and geometry, but it may be possible in some nonfavorable case to find appropriate modifications of the model which do not spoil its main feature (its linearity). For example, we have recently shown that in three-dimensional homogeneous turbulence with no mean flow, one can replace the local subfilter energy transfer by a mere turbulent viscosity (instead of dropping them) to obtain a model which reproduces all the mean and statistical properties of the complete Navier-Stokes equations.

\section{ACKNOWLEDGMENTS}

We thank M. Oberlack for many enlightening discussions about shear flow profiles and the meaning of algebraic laws. We also thank P. Sullivan for interesting comments on the manuscript. N.K.-R.K. would like to thank McMaster University and NSERC for research funding during this project. B.D. was partly supported by a NATO fellowship.

\section{APPENDIX A: FILTER AND AVERAGE}

In Appendices $\mathrm{A}-\mathrm{G}$, we present a number of technical results necessary in the derivation of our resolved and subfilter equations.

\section{Definition}

In order to distinguish between large and small scales we introduce a filter function $g(\mathbf{x})$. For reasons of convenience which will become clear in Sec. II B, we choose the following separable function:

$$
g(\mathbf{x})=\Pi_{i} g_{i}\left(x_{i}\right)
$$

where $g_{i}$ are positive functions, which are normalized to unity with a characteristic length-scale $l_{i}^{*}$. Thus, we can write them as

$$
g_{i}\left(x_{i}\right)=\frac{1}{l_{i}^{*}} \tilde{g}_{i}\left(\frac{x_{i}}{l_{i}^{*}}\right),
$$

where the $\tilde{g}_{i}$ are functions which decay rapidly when their argument is larger than one. The choice of the characteristic length-scales $l_{i}^{*}$ will be discussed further in Sec. III. Physically, this scale represents a scale intermediate between the scale of the resolved field, $L$, and the scale of the subfilter field $l$. In the sequel of the paper, we shall assume that the ratio $l / L$ is a small parameter, called the scale separation.

Note that the filter influences our results via constants which are filter dependent. We shall mention this dependence where appropriate. Using this filter, we can now decompose the velocity field into its mean (large-scale) and its fluctuating (small-scale) contribution,

$$
\begin{aligned}
& \mathbf{u}(\mathbf{x}, t)=\mathbf{U}(\mathbf{x}, t)+\mathbf{u}^{\prime}(\mathbf{x}, t), \\
& \mathbf{U}(\mathbf{x}, t) \equiv \bar{u}=\int g\left(\mathbf{x}-\mathbf{x}^{\prime}\right) \mathbf{u}\left(\mathbf{x}^{\prime}, t\right) d \mathbf{x}^{\prime} .
\end{aligned}
$$

Notice that our filtering only concerns spatial scales, not time scales. In experimental measurements, however, time and space filtering are often linked because of the use of the Taylor hypothesis. In this spirit, we allow the possibility of adding time filtering to our spatial (or scale) filtering. This freedom will be used in our analytical computations, to assume a $\delta$-correlation in time. We now summarize some salient properties of our filtering procedure.

\section{Properties}

Note that we employ scale filtering, and not a statistical filtering via the usual ensemble averaging. This scale filtering is in the spirit of the LES, where large and small scales are modeled separately in order to improve computational efficiency. In the present case, the separation between large and small scales is dictated by physical considerations (Sec. III) which only apply because of the scale difference (scale separation) between the two components. This means that our hypothesis might not be valid for ensemble averaging. This also means that we should be cautious when averaging, since for example the filtering of the product of two filtered quantities is not necessarily the product of the filtered quantities, $\overline{\bar{u} \bar{v}} \neq \overline{u v}$. This happens when the filter size cannot be chosen to be too small because of insufficient scale separation. Similarly, the filtering of a product of a small-scale quantity and a large-scale filtered quantity is not necessarily zero. As we shall see, this produces additional terms in the averaged equations with respect to the usual Reynolds average, based on ensemble averaging.

Another important difference between the LES filtering and the ensemble average is in the combination of derivatives and averaging; derivatives and averaging commute for ensemble averaging. For LES filtering, this is not the case in general. To see this, consider the average of some derived quantity, say $\partial_{x} u$, and integrate by parts over $x^{\prime}$. We get 


$$
\begin{aligned}
\overline{\partial_{x} u}= & \int g\left(\mathbf{x}-\mathbf{x}^{\prime}\right) \partial_{x^{\prime}} u\left(\mathbf{x}^{\prime}\right) d \mathbf{x}^{\prime} \\
= & \left.\int d y d z g\left(\mathbf{x}-\mathbf{x}^{\prime}\right) u\left(\mathbf{x}^{\prime}\right)\right|_{x^{\min }} ^{x_{\max }} \\
& -\int\left(\partial_{x^{\prime}} g\left(\mathbf{x}-\mathbf{x}^{\prime}\right)\right) u\left(\mathbf{x}^{\prime}\right) d \mathbf{x}^{\prime} .
\end{aligned}
$$

By symmetry, the derivative over $x^{\prime}$ in the second term of the rhs of (A4) can be changed into minus a derivative over $x$, which can then be taken out of the integral, giving a term $\partial_{x} \bar{u}$. We then see that average and derivative commute only if the surface term (the first term in the rhs) is zero. If not, this term has to be taken into account in the derivation of LES equations. When dealing with unbounded systems, this problem of commutativity is not important, since one usually considers physical quantities which tend to zero at infinity. The noncommutativity arises in finite size systems, where boundary conditions may reflect some physical processes. In plane parallel flows this is the case in the vertical direction at the bottom of the layer where we have to take into account the momentum and heat flux at the surface $z=0$.

Finally, we can use the scale separation $l / L \ll 1$ to estimate the average of resolved (or subfilter quantities) because the scale of $g$ is just intermediate between $l$ and $L$. So, it is large with respect to $l$, and small with respect to $L$. We then find, for a quantity $Q$ with average $\bar{Q}$ and subfilter part $Q^{\prime}$,

$$
\begin{aligned}
\bar{Q} & =\int g\left(\mathbf{x}-\mathbf{x}^{\prime}\right) \overline{Q\left(x^{\prime}\right)} d \mathbf{x}^{\prime} \\
& \approx \overline{Q(x)} \int g\left(\mathbf{x}-\mathbf{x}^{\prime}\right) d \mathbf{x}^{\prime}=\overline{Q(x)}, \\
\overline{Q^{\prime}} & =\int g\left(\mathbf{x}-\mathbf{x}^{\prime}\right) Q^{\prime}\left(x^{\prime}\right) d \mathbf{x}^{\prime} \approx 0 .
\end{aligned}
$$

Here, we have used the fact that $\bar{Q}$ is a slow function with respect to $g$ and can be taken out of the integral. In the same way, $g$ is a slow function with respect to $Q^{\prime}$ and can be taken out of the integral. This approximation is valid up to order $\epsilon_{*}=l^{*} / L \approx l / l^{*}$ (if we take $l^{*}$ as the geometrical mean of the large and small scales).

\section{APPENDIX B: GABOR TRANSFORM}

\section{Definition}

Our ultimate goal is to write an equation for the small (subfilter) scales, as a function of the large (resolved) scales. However, we would like to take into account the fact that the small scales are strongly inhomogeneous in space. A good way of representing inhomogeneous fields is to decompose them into wave packets, using for example, the Gabor transformation $^{36}$

$$
\hat{u}^{\prime}(\mathbf{x}, \mathbf{k}, t)=\int f\left(\mathbf{x}-\mathbf{x}^{\prime}\right) e^{i \mathbf{k} \cdot\left(\mathbf{x}-\mathbf{x}^{\prime}\right)} \mathbf{u}^{\prime}\left(\mathbf{x}^{\prime}, t\right) d \mathbf{x}^{\prime} .
$$

For reasons of convenience which will become clear later, we choose here $f(\mathbf{x})=\sqrt{g(\mathbf{x})}$ (since $g$ is a positive function; this is always possible). We could also have achieved the same decomposition using a wavelet transformation, ${ }^{37}$ but it is more complicated for analytical computations.

\section{Useful properties of the Gabor transform}

A few properties of the Gabor transformation (B1) are worth mentioning at this point. First, the inverse Gabor transform is easily obtained by an integration over $\mathbf{k}$,

$$
f(0) \mathbf{u}^{\prime}(\mathbf{x}, t)=\frac{1}{(2 \pi)^{D}} \int \hat{\mathbf{u}}^{\prime}(\mathbf{x}, \mathbf{k}, t) d \mathbf{k} .
$$

Second, we note that as in filtering, the commutation of Gabor transform and partial derivative is subject to surface terms which depend on the boundary conditions (for reasons analogous to those given in Appendix A 2). Also, there is an interesting approximation to the derivative of the Gabor transform,

$$
\partial_{i} \hat{\mathbf{u}}^{\prime} \approx i k_{i} \hat{\mathbf{u}}^{\prime}+O(1 /(L k)),
$$

where $L$ is the typical scale of the large-scale flow. Technically speaking, $\boldsymbol{\epsilon}=1 /(k L)$ labels the scale separation between the large scales and the small scales. It is a parameter less than one. Third, it is interesting to consider the Gabor transform of a quantity involving the product of a function varying over large scales (e.g., U) and a function varying over small scales (e.g., $\mathbf{u}^{\prime}$ ),

$$
\widehat{\mathbf{U u}^{\prime}}=\int f\left(\mathbf{x}-\mathbf{x}^{\prime}\right) e^{i \mathbf{k} \cdot\left(\mathbf{x}-\mathbf{x}^{\prime}\right)} \mathbf{U}\left(\mathbf{x}^{\prime}, t\right) \mathbf{u}^{\prime}\left(\mathbf{x}^{\prime}, t\right) d \mathbf{x}^{\prime} .
$$

Because the kernel $f$ varies over scales of the order of $1 / k_{c}$, while $U$ varies over scales of the order $L$, one can Taylor expand the function $U$ around the point $\mathbf{x}^{\prime}$. To first order in $\epsilon$, and after integration by parts, we obtain

$$
\widehat{\mathbf{U u}^{\prime}}(\mathbf{x}, \mathbf{k}, t)=\mathbf{U}(\mathbf{x}, t) \hat{\mathbf{u}}^{\prime}+i \nabla_{i}\left(U_{j}(\mathbf{x}, t)\right) \nabla_{k_{i}} \hat{\mathbf{u}}^{\prime} .
$$

This expansion will be useful in our derivations.

Finally, it is interesting to consider the average of the product of two small-scale quantities, e.g., the Reynolds stress $\overline{u_{i}^{\prime} u_{j}^{\prime}}$. Using our definition of filtering (A3) and the fact that $(2 \pi)^{D} \delta\left(x^{\prime}-x^{\prime \prime}\right)=\int e^{i k\left(x-x^{\prime}\right)} e^{-i k\left(x-x^{\prime \prime}\right)} d k$ and $f^{2}$ $=g$, we can write the Reynolds stress at point $\mathbf{x}$ and time $t$ as a function of the Gabor transform,

$$
\begin{aligned}
\overline{u_{i}^{\prime} u_{j}^{\prime}}= & \frac{1}{2(2 \pi)^{D}} \int\left(\hat{u}_{i}^{\prime}(\mathbf{k}, \mathbf{x}, t) \hat{u}_{j}^{\prime}(-\mathbf{k}, \mathbf{x}, t)\right. \\
& \left.+\hat{u}_{i}^{\prime}(-\mathbf{k}, \mathbf{x}, t) \hat{u}_{j}^{\prime}(\mathbf{k}, \mathbf{x}, t)\right) d \mathbf{k} .
\end{aligned}
$$

\section{APPENDIX C: SPECTRA AND CHOICE OF THE CUTOFFS}

The definition of our filtering involves the choice of the characteristic length scale of the filter. To choose this scale in a physically meaningful way we can use the spectral measurements of Ref. 22. Their result indicates that the streamwise and vertical velocity components of a wall-bounded turbulent flow have different spectral characteristics. Both are characterized by a Kolmogorov energy spectrum at small 
scales $\left(E(k) \sim k_{x}^{-5 / 3}\right)$, and by an approximately flat spectrum at large scales. However, the wave number of the transition between the two regions is approximately one order of magnitude larger for the vertical component than for the streamwise component [where an additional transition region where $E(k) \sim k_{x}^{-1}$ is present over one decade before the spectrum flattens]. These features suggest choosing the filtering scale to be the scale corresponding to the peak wave number of the vertical velocity spectrum. In this way, we can expect the resolved vertical velocity to be smaller than the streamwise resolved velocity, enabling a substantial simplification of the resolved and subfilter-scale dynamics. Since this scale will nevertheless correspond to an inertial range scale of the streamwise velocity spectrum, the subfilter streamwise velocities should still be much smaller than the resolved streamwise velocities. This guarantees that the dynamics between small and large scales will be essentially nonlocal (i.e., between resolved and subfilter scales only) at the subfilter level, allowing for a linearization of the small-scale dynamics, and, therefore, analytical solutions.

\section{APPENDIX D: CORRELATION BETWEEN FORCES AND RESOLVED VELOCITY}

In this appendix, we compute the correlation between the Gabor transforms of resolved velocity and forces, using the $\delta$-correlation assumption. Using definition of GT we have

$$
\begin{aligned}
\left\langle\hat{U}\left(\boldsymbol{x}_{1}, \boldsymbol{k}_{1}, t_{1}\right) \hat{F}_{m}^{\perp}\left(\boldsymbol{x}_{2}, \boldsymbol{k}_{2}, t_{2}\right)\right\rangle & \\
= & \int f\left(\boldsymbol{\epsilon}^{*}\left|\boldsymbol{x}_{1}-\boldsymbol{x}^{\prime}\right|\right) f\left(\boldsymbol{\epsilon}^{*}\left|\boldsymbol{x}_{2}-\boldsymbol{x}^{\prime \prime}\right|\right) e^{i k_{1} \cdot\left(x_{1}-\boldsymbol{x}^{\prime}\right)+i \boldsymbol{k}_{2} \cdot\left(\boldsymbol{x}_{2}-\boldsymbol{x}^{\prime \prime}\right)} \\
& \times\left\langle U\left(\boldsymbol{x}^{\prime}, t_{1}\right) F_{m}^{\perp}\left(\boldsymbol{x}^{\prime \prime}, t_{2}\right)\right\rangle \mathrm{d} \boldsymbol{x}^{\prime} \mathrm{d} \boldsymbol{x}^{\prime \prime} \\
= & \int f^{2}\left(\boldsymbol{\epsilon}^{*}\left|\boldsymbol{x}_{1}-\boldsymbol{x}^{\prime}\right|\right) e^{i \boldsymbol{k}_{1} \cdot\left(\boldsymbol{x}_{1}-\boldsymbol{x}^{\prime}\right)+i \boldsymbol{k}_{2} \cdot\left(\boldsymbol{x}_{2}-\boldsymbol{x}^{\prime \prime}\right)} \\
& \times U\left(\mathbf{x}^{\prime}, t_{1}\right) F_{m}^{\perp}\left(\mathbf{x}^{\prime \prime}, t_{2}\right) \delta\left(\mathbf{x}^{\prime}-\mathbf{x}^{\prime \prime}\right) \delta\left(t_{1}-t_{2}\right) \mathrm{d} \mathbf{x}^{\prime} \mathrm{d} \mathbf{x}^{\prime \prime} \\
= & U\left(\boldsymbol{x}_{1}, t_{1}\right) f^{2}\left(\boldsymbol{\epsilon}^{*}\left|\boldsymbol{x}_{1}-\boldsymbol{x}_{2}\right| / 2\right) \delta\left(t_{1}-t_{2}\right) e^{\left.i \boldsymbol{k}_{2} \cdot \boldsymbol{x}_{2}+i \boldsymbol{k}_{1} \cdot \boldsymbol{x}_{1}\right)} \\
& \times \mathrm{FT}\left(F_{m}^{\perp}\right)(\boldsymbol{k} 1+\boldsymbol{k} 2),
\end{aligned}
$$

where FT is the Fourier transform. When deriving (D1) we took into account that $F^{\perp}$ decays faster in $\boldsymbol{r}$ than $f$ does (we put $\mathbf{x}^{\prime}=\mathbf{x}^{\prime \prime}$ in $f$ ). We also took into account that $U$ is a slow function so that it can be taken out of the integral by setting $\mathbf{x}^{\prime}=\mathbf{x}_{1}$.

\section{APPENDIX E: ANALYTICAL SOLUTIONS OF SMALL- SCALE EQUATION}

In this appendix, we provide the analytical solution to the system of ray Eqs. (22). To solve this system of equations, we first solve the homogeneous system (i.e., with $F_{u}$ $\left.=F_{v}=F_{w}=0\right)$. First, we note that by introducing

$$
\begin{aligned}
& \left(u^{\dagger}, v^{\dagger}, w^{\dagger}\right)=(\hat{u}, \hat{v}, \hat{w}) D, \\
& D=\exp \left(\frac{\nu k^{2}}{\partial_{z} U}\left(R+R^{3} / 3\right)\right),
\end{aligned}
$$

we can eliminate the viscous terms from the homogeneous equations. The ray equations can then be integrated in the standard way, starting from $\hat{w}$. We find three independent solutions to the homogeneous system, two trivial ones,

$$
\begin{aligned}
& \hat{w}_{1}=\hat{v}_{1}=0, \quad \hat{u}_{1}=D, \\
& \hat{w}_{3}=\hat{u}_{3}=0, \quad \hat{v}_{3}=D,
\end{aligned}
$$

and a more complicated one,

$$
\begin{aligned}
& \frac{\hat{u}_{2}}{D}=-\cos \theta \frac{R}{1+R^{2}}+\frac{\sin ^{2} \theta}{\cos \theta} \operatorname{atan} R, \\
& \frac{\hat{v}_{2}}{D}=-\sin \theta\left(\frac{R}{1+R^{2}}+\operatorname{atan} R\right), \\
& \frac{\hat{w}_{2}}{D}=\frac{1}{1+R^{2}} .
\end{aligned}
$$

Now, we introduce the $3 \times 3$ matrix $K(R)$ whose columns are made up of the three independent solutions. We also introduce the vector $\mathbf{F}=\left(\partial_{z} U \cos \theta\right)^{-1}\left(F_{u}^{\perp}, F_{v}^{\perp}, F_{w}^{\perp}\right)$. The general solution of the inhomogeneous system (22) can then be written $\phi(R)=(\hat{u}, \hat{v}, \hat{w})$, with

$$
\phi(R)=K(R) K^{-1}\left(R_{0}\right) \phi_{0}+K(R) \int_{R_{0}}^{R} K^{-1}(r) \mathbf{F}(r) d r .
$$

Taking as initial condition $\phi=0$ at $R_{0}=-\infty$ (no smallscales at $t=-\infty)$, we can finally write the general solution as

$$
\phi(R)=\int_{-\infty}^{R} K(R) K^{-1}(r) \mathbf{F}(r) d r
$$

Note that the integrand is just the solution of the homogeneous system, with initial condition $\phi(R=r)=\mathbf{F}(r)$. This point is used in Sec. IV. Also, consider a product of two components of $\phi$, say $\phi_{u} \phi_{w}^{*}=\hat{u} \hat{w}^{*}$. Upon using (E5), and the definition of the average using Gabor transform (B6), we can write

$$
\begin{aligned}
\langle\overline{u w}\rangle= & \sum_{j, l, j^{\prime}, l^{\prime}=1}^{2} \sum d \theta k_{h} d k_{h} d R \int_{-\infty}^{R} d r_{0} \\
& \times \int_{-\infty}^{R} d r_{1} K_{1 j}(R) K_{1 j^{\prime}}^{*}(R) K_{j l}\left(r_{0}\right) K_{j^{\prime} l^{\prime}}^{*}\left(r_{1}\right) \\
& \times\left\langle F_{l}\left(r_{0}\right) F_{l^{\prime}}^{*}\left(r_{1}\right)\right\rangle .
\end{aligned}
$$

Because of the delta correlations of the force (see Ref. 14), the average brings down a factor $-\cos \theta \partial_{z} U \tau \delta\left(r_{1}-r_{0}\right)$ [because $\left.t-t_{0}=-\cos \theta \partial_{z} U\left(R-R_{0}\right)\right]$, where $\tau$ is a typical time scale (necessary for dimensional reasons). So after integration over say, $r_{1}$, (E6) becomes 


$$
\begin{aligned}
\langle\overline{u w}\rangle= & -\partial_{z} U \tau \int \cos \theta d \theta k_{h} d k_{h} d R \int_{-\infty}^{R} d r_{0} k \\
& \times \sum_{j, l, j^{\prime}, l^{\prime}=1}^{2} K_{1 j}(R) K_{1 j^{\prime}}^{*}(R) K_{j l}\left(r_{0}\right) K_{j^{\prime} l^{\prime}}^{*}\left(r_{1}\right) \\
& \times\left\langle F_{l}\left(r_{0}\right) F_{l^{\prime}}^{*}\left(r_{0}\right)\right\rangle .
\end{aligned}
$$

Here, the integrand of (E7) is just the product of two solutions of the homogeneous system with initial condition $\phi(R=r)=\mathbf{F}(r)$. This property is also used in Sec. IV. We stress once again that this simplification is only valid when one performs the averaging procedure.

\section{APPENDIX F: DIVERGENCES}

The expression (26) for $\langle\overline{U w}\rangle$ involves a divergence in the inviscid limit, which can be used to study the asymptotic expansion of $\tilde{\eta}$. The divergence actually occurs at $\theta$ $= \pm \pi / 2$ due to the inverse cosine factor. To study it, we use symmetries of the forcing to write the $\theta$ dependent part of the integral as

$$
\begin{gathered}
\int_{-\pi}^{\pi} d \theta \frac{\mathrm{FT}\left(F_{w}^{\perp}\right)}{\cos \theta} e^{i k_{h} \cos \theta\left(R+R_{0}\right)} e^{-\nu k_{h}^{2}} \int_{R_{0}}^{R}\left(1+X^{2}\right) d X / \cos \theta \partial_{z} U \\
\quad=4 \int_{0}^{\pi / 2} d \theta \frac{\mathrm{FT}\left(F_{w}^{\perp}\right)}{\cos \theta} e^{i k_{h} \cos \theta\left(R+R_{0}\right)} e^{-\alpha / \cos \theta}
\end{gathered}
$$

where $\alpha=\nu k_{h}^{2} \int_{R_{0}}^{R}\left(1+X^{2}\right) d X / \partial_{z} U$ will be our small parameter in the limit $\nu \rightarrow 0$ (see Ref. 14). A change of variables $\cos \theta \rightarrow s$ allows us to write the integral involved in $(\mathrm{F} 1)$ as

$$
\begin{aligned}
& 4 \int_{0}^{1}\left(\frac{\mathrm{FT}\left(F_{w}^{\perp}\right)(s) e^{i k_{h} s\left(R+R_{0}\right)}}{s \sqrt{1-s^{2}}}-\frac{\mathrm{FT}\left(F_{w}^{\perp}\right)(0)}{s}\right) e^{-\alpha / s} \\
& +4 \int_{0}^{1} \frac{\mathrm{FT}\left(F_{w}^{\perp}\right)(0)}{s} e^{-\alpha / s} .
\end{aligned}
$$

The first integral is convergent at $s=0$, and its leading-order approximation can be easily found by expanding the exponential. The second integral is proportional to the exponential integral function $E_{i}(x)=\int_{0}^{1} e^{-t / x} d t / t$, whose expansion near $x=0$ is

$$
E_{i}(x)=C+\ln x+O(x),
$$

where $C$ is Euler's constant. Overall, the expansion for $\langle\overline{U w}\rangle$ is

$$
\begin{aligned}
& \langle\overline{U w}\rangle=\eta \frac{U}{\partial_{z} U}, \\
& \eta=\eta_{0} \ln \alpha+\eta_{1}+O(\alpha), \\
& \eta_{0}=-4 \int_{0}^{\infty} k_{h} d k_{h} \int_{-\infty}^{\infty} d R \int_{-\infty}^{R} F_{w}^{\perp}\left(0, k_{h}, k_{h} R_{0}\right) \frac{1+R_{0}^{2}}{1+R^{2}},
\end{aligned}
$$

$$
\begin{aligned}
\eta_{1}= & -4 \int_{0}^{\infty} k_{h} d k_{h} \int_{-\infty}^{\infty} d R \int_{-\infty}^{R} \frac{1+R_{0}^{2}}{1+R^{2}} \int_{0}^{1} d s \\
& \times\left[C \operatorname{FT}\left(F_{w}^{\perp}\right)\left(0, k_{h}, k_{h} R_{0}\right)\right. \\
& +\frac{1}{s}\left(\frac{\mathrm{FT}\left(F_{w}^{\perp}\right)\left(k_{h} s, k_{h} \sqrt{\left.\left(1-s^{2}\right), k_{h} R_{0}\right) e^{k_{h} s\left(R_{0}+R\right) z}}\right.}{\sqrt{1-s^{2}}}\right. \\
& \left.\left.-F_{w}^{\perp}\left(0, k_{h}, k_{h} R_{0}\right)\right)\right] .
\end{aligned}
$$

In the isotropic case [when $F_{w}^{\perp}$ only depends on $k_{h}(1$ $\left.+R_{0}^{2}\right)$ ], symmetries of the integral defining $\eta_{0}$ make it equal to 0 .

\section{APPENDIX G: ANALYTICAL EXPRESSION OF FUNCTIONS USED TO FIT THE DATA}

Two functions $H(z)$ and $\eta(z)$ are used to fit the data for each comparison. We give the analytical expression of these functions for each of the three cases.

\section{Function $\boldsymbol{H}_{\mathrm{eff}}$ or $\boldsymbol{H}$}

$H_{\text {eff }}$ is computed from numerical integration of $H$ $=\int d h / d z d z$, where

$$
\frac{d H}{d z}=\frac{d H_{w}+d H_{c}}{2} f(z)+\frac{d H_{w}-d H_{c}}{2},
$$

where $f(z)$ is a smooth function so that

$$
\begin{aligned}
& f=+1 \text { for } z_{w}<z<z_{t} \quad \text { (near wall), } \\
& f=-1 \text { for } z_{t}<z<z_{c} \quad \text { (core region), }
\end{aligned}
$$

and the integration constant is chosen so that

$$
H\left(z_{w}\right)=\frac{-K R_{*}}{1+K R_{*}} .
$$

The expression of the slopes $\left(d H_{w}\right.$ and $\left.d H_{c}\right)$ are given in Table I. We have chosen the following smooth function $f$ :

$$
f(z)=\tanh \left[\frac{z-z_{t}}{5 * z_{t}}\right] \text {. }
$$

\section{Function $\eta_{\text {eff }}$ or $\boldsymbol{\eta}$}

This function is chosen so that

$$
\eta=\frac{\eta_{w}+\eta_{c}}{2} f(z)+\frac{\eta_{w}-\eta_{c}}{2}
$$

where $f(z)$ is the same smooth function than for $H$. The expression of the constants $\eta_{w}$ and $\eta_{c}$ is given in Table I.

\section{Table of fitted parameters}

The fitted parameters are, in principle, $R_{*}, K, \alpha, U_{\infty}$, $\eta_{0}$, and $z_{t}$. For simplification, we only fitted $K$ on the Zagarola data, and fixed $K=0.45(k=0.44)$ in the channel flow (boundary layer), and the value of $U_{\infty}$. 
${ }^{1}$ J. O. Hinze, Turbulence, 2nd ed. (McGraw-Hill, New York, 1975).

${ }^{2}$ K. S. Yajnik, "Asymptotic theory of turbulent shear flows," J. Fluid Mech. 42, 411 (1970)

${ }^{3}$ G. L. Mellor, "The large Reynolds number asymptotic theory of turbulent boundary layers," Int. J. Eng. Sci. 10, 851 (1972).

${ }^{4}$ G. I. Barenblatt, "Scaling laws for fully developed turbulent shear flow. Part 1. Basic hypotheses and analysis," J. Fluid Mech. 248, 513 (1993).

${ }^{5}$ J. Nikuradse, "Gesetzmässigkeiten der turbulenten Strömung in glatten Röhren," Forsch. Arb. Ing-Wes No. 356, 1932 (English translation NACA TT F-10, p. 359)

${ }^{6}$ M. V. Zagarola, A. E. Perry, and A. J. Smits, "Mean-flow scaling of turbulent pipe flow,", Phys. Fluids 9, 2094 (1997).

${ }^{7}$ W. K. George, L. Castillo, and M. Wosnik, "A theory for turbulent pipe and channel flows," TAM Report No. 872, 1997.

${ }^{8} \mathrm{~W}$. K. George and L. Castillo, "The zero pressure-gradient turbulent boundary layer,"' Appl. Mech. Rev. 50, 689 (1997).

${ }^{9}$ M. Oberlack, "A unified approach for symmetries in plane parallel turbulent shear flows," J. Fluid Mech. 427, 299 (2001).

${ }^{10}$ G. L. Niederschulte, "Turbulent flow through a rectangular channel," Ph.D. thesis, University of Illinois, Department of Theoretical and Applied Mechanics, 1996.

${ }^{11}$ M. V. Zagarola, "Mean-flow scaling of turbulent pipe flow," Ph.D. dissertation, Princeton University, 1996.

${ }^{12}$ M. Oberack, "Similarity in nonrotating and rotating turbulent pipe flows," J. Fluid Mech. 379, 1 (1999).

${ }^{13}$ S. Chen, C. Foias, D. D. Holm, E. Olson, E. S. Titi, and S. Wynne, "Comasa-Holm equations as a closure model for turbulent channel and pipe flow," Phys. Rev. Lett. 81, 5338 (1998).

${ }^{14}$ S. Nazarenko, N. Kevlahan, and B. Dubrulle, "Nonlinear RDT theory of near wall turbulence," Physica D 139, 158 (2000).

${ }^{15}$ S. Nazarenko, "On exact solutions for near-wall turbulence theory," Phys. Lett. A 264, 444 (2000).

${ }^{16}$ J-P. Laval, B. Dubrulle, and S. Nazarenko, "Nonlocality and intermittency in 3D turbulence," Phys. Fluids 13, 1995 (2001).

${ }^{17}$ J. A. Domaradzki, W. Liu, C. Härtel, and L. Kleiser, "Energy transfer in numerically simulated wall-bounded turbulent flows," Phys. Fluids 6, 1583 (1994)

${ }^{18}$ J. Carlier, J-P. Laval, J. M. Foucaut, and M. Stanislas, "Some experimental support at large Reynolds number to a new hypothesis for turbulence modeling," C. R. Acad. Sci., Ser. IIb: Mec., Phys., Chim., Astron. 329, 1 (2001).

${ }^{19}$ A. A. Townsend, "The structure of turbulent shear flow," in The Structure of Turbulent Shear Flow, 2nd ed. (Cambridge University Press, Cambridge, 1976).

${ }^{20}$ F. Hersant, B. Dubrulle, and M. Wang, "Nonlocality of interactions in a turbulent shear flow,'” CTR Report No. 2000.
${ }^{21}$ J-P. Laval, B. Dubrulle, and S. Nazarenko, "Nonlocality of interaction of scales in the dynamics of 2D incompressible fluids," Phys. Rev. Lett. 83, 4061 (1999).

${ }^{22}$ A. E. Perry, S. Henbest, and M. S. Chong, "A theoretical and experimental study of wall turbulence," J. Fluid Mech. 165, 163 (1986).

${ }^{23}$ J. A. Domaradzki and E. M. Saiki, "A subgrid-scale model based on the estimation of unresolved scales of turbulence," Phys. Fluids A 9, 2148 (1997).

${ }^{24}$ J. Bardina, J. H. Ferziger, and W. C. Reynolds, "Improved subgrid models for large eddy simulation," AIAA Paper No. 80-1357, 1980.

${ }^{25}$ J. Kestin and P. D. Richardson, "Heat transfer across turbulent, incompressible boundary layers,' Int. J. Heat Mass Transf. 6, 147 (1963).

${ }^{26}$ R. D. Moser, J. Kim, and N. N. Mansour, "Direct numerical simulation of turbulent channel flows up to $\operatorname{Re}_{\tau}=590$," Phys. Fluids 11, 943 (1999).

${ }^{27}$ M. Nockemann, M. Schober, J. Bruns, R. Abstient, and D. W. Eckert (DNW), "Vermessung der wangrenzschicht im Deutsch-Niederländischen windkanal bei hohen Reynoldszahlen," Abhln. Aerodynamisches Institut RWTH Aachen Heft 32, 1994.

${ }^{28}$ B. Dubrulle and U. Frisch, "Eddy viscosity of parity-invariant flow," Phys. Rev. A 43, 5355 (1991).

${ }^{29}$ B. F. Farrell and P. J. Ioannou, "Optimal excitation of three-dimensional perturbations in viscous constant shear flow," Phys. Fluids A 5, 1390 (1993).

${ }^{30}$ T. Cebeci and A. M. O. Smith, Analysis of Turbulent Boundary Layers (Academic, New York, 1974).

${ }^{31}$ J. C. R. Hunt, D. D. Stretch, and R. E. Britter, Proceedings of the IMA Conference on Stability Stratified Flow and Dense Gas Dispersion, edited by J. S. Puttock (Clarendon, Oxford, 1988).

${ }^{32}$ B. Dubrulle and S. Nazarenko, "Interaction of turbulence and large-scale vortices in incompressible 2D fluid," Physica D 110, 123 (1998).

${ }^{33}$ B. Dubrulle, J-P. Laval, and P. Sullivan, "A new dynamical subgrid model for the planetary surface layer. I. The model,' J. Atmos. Sci. (submitted).

${ }^{34}$ B. Dubrulle, J-P. Laval, P. Sullivan, and J. Werne, “A new dynamical subgrid model for the planetary surface layer. II. Analytical computation of fluxes, mean profiles, and variances," J. Atmos. Sci. (submitted).

${ }^{35}$ E-J. Kim and B. Dubrulle, "Are the energy cascades direct or inverse in MHD turbulence?', Physica D (submitted).

${ }^{36}$ S. Nazarenko, N. Kevlahan, and B. Dubrulle, "Semiclassical theory for rapid distortion of homogeneous turbulence," J. Fluid Mech. 390, 325 (1999).

${ }^{37}$ A. Arneodo, J-F. Muzy, and S. Roux, "Experimental analysis of selfsimilarity and random cascade process: application to fully developed turbulence data,"' J. Phys. II 7, 363 (1997). 